\title{
Crossovers in Unitary Fermi Systems
}

\author{
Henning Heiselberg
}

\begin{abstract}
Universality and crossover is described for attractive and repulsive interactions where, respectively, the BCS-BEC crossover takes place and a ferromagnetic phase transition is claimed. Crossovers are also described for optical lattices and multicomponent systems. The crossovers, universal parameters and phase transitions are described within the Leggett and NSR models and calculated in detail within the Jastrow-Slater approximation. The physics of ultracold Fermi atoms is applied to neutron, nuclear and quark matter, nuclei and electrons in solids whenever possible. Specifically, the differences between optical lattices and cuprates is discussed w.r.t. antiferromagnetic, d-wave superfluid phases and phase separation.
\end{abstract}

\section{Introduction and the Bertsch problem}

A decade ago at the 10th Manybody Conference G. Bertsch posed the problem (see [1] for full text):

How does a system of Fermi particles with infinite s-wave scattering length but vanishing interaction range behave?

This seemingly innocent question turned out to be rather fundamental and triggered an explosion of interest and number of papers on physics now referred to as universal physics, the unitarity limit, BCS-BEC crossover, and strongly interacting and correlated systems in general. His question was partly motivated from nuclear physics and the physics of neutron stars where dilute gases of neutrons exist in the inner crust. The neutron-neutron ${ }^{1} S_{0}$ scattering length $a \simeq-18.5 \mathrm{fm}$ is long (and negative) compared to the order of magnitude smaller interaction range $R \sim 1 \mathrm{fm}$,

Henning Heiselberg

Applied Research, DALO, Lautrupbjerg 1-5, DK-2750 Ballerup, Denmark, e-mail:hh@mil.dk 
because two neutrons almost have a bound state. Admittably, Bertsch was also motivated by expectations that cold gases of Fermi atoms might be created like the BEC a few years earlier, and that it might be possible to tune interactions near Feshbach resonances between two hyperfine states in order to make the scattering length truly go to $\pm \infty$. Both were rapidly and successfully accomplished in a number of remarkable experiments where also the BCS-BEC crossover, multicomponent systems, optical lattices, etc. have been studied.

The solution to the Bertsch problem for two-component systems is as simple as it is fundamental [2, 3]:

Since $R \rightarrow 0$ and $a \rightarrow \pm \infty$ both parameters must vanish from the problem leaving only one remaining length scale: the interparticle distance or equivalently the inverse of the Fermi wavenumber $k_{F}^{-1}$. All thermodynamic quantities become universal.

The proof is a dimensional argument which will be described in detail below. As an example, the energy per particle is an universal constant times the energy of a non-interacting Fermi gas $(3 / 5) E_{F}=3 \hbar^{2} k_{F}^{2} / 10 m$. The $a \rightarrow \pm \infty$ limit is referred to as the universal or unitarity limit and the gas as a unitary gas. The name comes from scattering theory where e.g. the s-wave scattering cross section at relative momentum $k$ is limited by $\sigma_{0} \leq 4 \pi / k^{2}$ due to unitarity of the scattering matrix. For weak interactions $\sigma_{0}=4 \pi a^{2}$ which would diverge like the energy per particle (see Eq. (3) - if extrapolated to $a \rightarrow \pm \infty$. In the unitary gas the cross section and the thermodynamic quantities are instead limited by unitarity and universality respectively.

In the neutron gas $|a|$ is long but fixed and the unitarity limit is defined as $|a| \gg r_{0} \gg R$, where $r_{0}=(3 n / 4 \pi)^{-1 / 3} \sim k_{F}^{-1}$ is the interparticle distance, $k_{F}$ the Fermi wavenumber at density $n=k_{F}^{3} / 3 \pi^{2}$ for two components/spin states. Thus the unitarity limit ranges from nuclear saturation density $n_{N M} \simeq 3 / 4 \pi R^{3} \simeq 0.15 \mathrm{fm}^{-3}$, and more than three orders of magnitude down in density to $\sim|a|^{-3}$. In this region, e.g. the energy per particle scales as an universal constant times $E_{F}$. The unitarity limit became easily accessible experimentally as it became possible to tune atomic interaction strengths near Feshbach resonances [4], where $a \rightarrow \pm \infty$ corresponding to two-atom bound states at threshold. The scattering length could also be extended to positive values corresponding to bound molecular states and for strongly bound states $a \rightarrow 0_{+}$even a molecular BEC.

The Bertsch problem described above was originally intended for two component system as spin 1/2 neutrons or ultracold atoms in two hyperfine states but it is also relevant for a nucleon gas, since the neutron and a proton have the weakly bound state of deuterium and therefore a large positive scattering length $a \simeq+20 \mathrm{fm}$. Isospin symmetric nuclear matter, however, has two spin and two isospin states i.e. four components and does not have a unitarity limit. It is in fact unstable at subnuclear matter densities where a liquid gas phase transition occurs. This was successfully described in [3] where estimates showed that as Pauli blocking between same spins is effectively reduced as the number of spin states increases, the Pauli pres- 
sure can no longer overcome the unitary attraction. Only two component systems are stable and provides a unitary gas. Three component systems are marginal and four and higher component systems are unstable. Such multicomponent systems are now studied, e.g. ${ }^{6} \mathrm{Li}$ with three hyperfine states [5], ${ }^{137} \mathrm{Yb}$ with six nuclear spin states [6], and heteronuclear mixtures of ${ }^{40} \mathrm{~K}$ and ${ }^{6} \mathrm{Li}$ [7]. Multi-component systems have intriguing similarities with neutron, nuclear and quark matter. In the latter color superconductivity between the 2 spin, 8 color and 2-3 flavor states may occur [8].

When the interactions are varied, e.g. near a Feshbach resonance, the thermodynamic quantities depend on the dimensionless parameter $a k_{F}$. All the thermodynamic quantities become universal functions of the crossover parameter $a k_{F}$ or equivalently $x=1 / a k_{F}$ in the sense that they do not depend on the system whether a gas of atoms, neutrons, or any other Fermi particle as long as $R \ll|a|, r_{0}$. The universality argument is intimately connected to the smooth approach to the unitarity limit and crossover. At finite temperatures the universal thermodynamic functions depend on $T / E_{F}$ as well. A decade earlier pairing models [9, 10, 11] had already described the crossover from BCS to the BEC limit and calculated pairing gaps, transition temperatures and chemical potentials.

In these lecture notes universality, crossover and correlations will be described. Not only the BCS-BEC crossover in uniform system but also the repulsive "ferromagnetic" crossover, in multicomponent systems, traps and lattices. Mostly Fermi atoms are discussed but applications to neutron, nuclear and quark matter, nuclei and electrons in solids are made wherever possible.

\section{Universality and Crossover}

Universality is usually understood as the universal scaling in the unitarity (Bertsch) limit where the interaction range $(R \rightarrow 0)$ and scattering length $(a \rightarrow \pm \infty)$ are sufficiently short and large respectively that the thermodynamic quantities are independent of either or any other details of the interaction (such as effective ranges, etc.) and only given by universal constants.

When only the interaction range is negligible $(R \rightarrow 0)$ but the scattering length varies, as it is possible in ultracold atomic systems around Feshbach resonances, there is another dimensionless parameter in the problem usually taken as $a k_{F}$ or $x=$ $1 / a k_{F}$. The thermodynamic quantities are thus universal functions of this crossover parameter varying from weakly interacting $x \rightarrow-\infty$ over the unitarity limit $x=$ 0 to the strongly interacting molecular limit $x \rightarrow+\infty$. In pairing models [10, 11] these limits are named the BCS and BEC limits respectively and the crossover as BCS-BEC crossover. When finite temperatures are considered the thermodynamic quantities are universal functions of both $x$ and $T / E_{F}$ [12].

It should be emphasized that there are several crossovers (see Fig. (1). The most common one is that for increasingly attractive scattering lengths also known from pairing models [10, 11] and referred to as the BCS-BEC crossover. Here the cold atoms start out in the weakly attractive limit with BCS pairing i.e. $x=1 / a k_{F} \rightarrow-\infty$ 

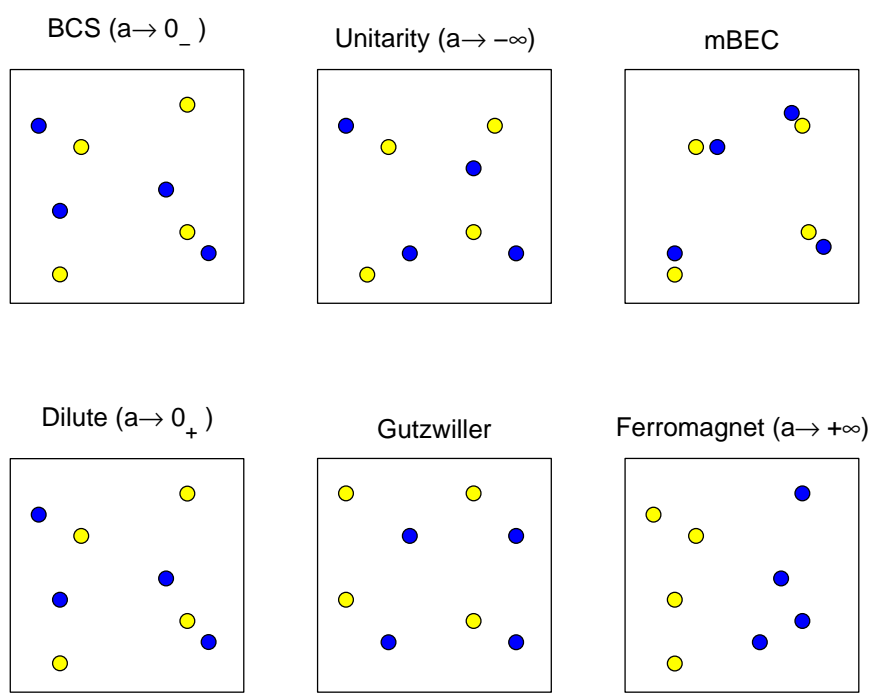

Fig. 1 Sketch of correlations in a two-component gas. Top row shows the gradual pairing of different components (spin states) with increasing attraction, i.e. the crossover from BCS via unitary gas to the molecular BEC. Bottow row shows the crossover for repulsive interactions from the dilute gas to either a hard sphere (Gutzwiller) or a ferromagnetic phase (see text).

and goes over the unitarity limit $x=0$ to the strongly attractive limit $x \rightarrow+\infty$, where a molecular BEC forms. Another crossover starts out with weak repulsive interactions, i.e. a small positive scattering length, and increasing it towards $a k_{F} \rightarrow+\infty$. A recent experiment claims to observe a phase transition in a ${ }^{6} \mathrm{Li}$ gas to a ferromagnetic state for $a k_{F} \gtrsim 2$ [13]. These two crossover are different because the latter has a node in the short range correlation function [22]. In principle there are a novel crossover for each number of nodes, however, they become increasingly short lived due to three-body losses. The different crossovers have different universal thermodynamic functions, universal parameters and quite different superfluid and ferromagnetic phases.

We will in the following subsections describe universality and crossover mainly for the BCS-BEC crossover and briefly for the "ferromagnetic" crossover. A number of thermodynamic functions are described which are calculated within the JastrowSlater approximation for the many-body wave function. 


\subsection{Universality in the unitarity limit}

The dimensional argument implies that any thermodynamic function, for example the energy per particle in units of the Fermi energy, can at zero temperature only depend on one dimensionless scale such as $x$

$$
\frac{E}{N}=\frac{3}{5} E_{F}(1+\beta(x))
$$

Here, the ratio between the interaction and kinetic energy $\beta(x)$ is a "universal" many-body function in sense that it and all other thermodynamic functions are independent of the system, e.g. dilute neutron matter, helium gases, atomic gases, etc. Earlier definitions are $\eta=(3 / 5)(1+\beta)[14]$ and $v=(5 / 3) \beta[3]$.

The universality and crossover hypothesis implicitly assumes that the transition to the unitarity limit and the crossover is smooth, i.e. the derivatives of $\beta(x)$ are finite. Taking the derivative with respect to density gives

$$
\frac{\partial E / N}{\partial n}=\frac{3}{5}(1+\beta(x)) \frac{\partial E_{G}}{\partial n}-\frac{E_{F}}{5 n} x \beta^{\prime}(x)
$$

where $\beta^{\prime}(x)=\partial \beta(x) / \partial x$. We observe that derivatives of $\beta$ w.r.t. densities or $k_{F}$ : $k_{F} \partial / \partial k_{F}=-x \partial / \partial x$, always brings a factor $x$ or $1 / a$. In the unitarity limit $x=0$, such terms as the second term in Eq. (2) therefore vanishes and only the universal

parameter $\beta(0)$ remains. By repeating the argument all density derivatives of the energy such as chemical potential, pressure, first sound speed, compressibility, etc. depend only on this one universal parameter $\beta(0)$. Other thermodynamic variables than density derivatives of $E / N$ can, however, depend on higher derivatives $\beta^{(n)}(0)$ as we shall see below.

The first estimates based on Pade' approximants and Galitskii resummation gave $\beta(0) \simeq-0.67[2,3]$ in the unitary limit whereas the Jastrow-Slater approximation yielded $\beta(0) \simeq-0.54$. The Leggett pairing model, which will be described in detail in a later section, predicts $\beta(0) \simeq-0.40$. Ground state energies have been calculated more accurately numerically by Monte Carlo for systems with a finite number of Fermions in a box. The earliest actually use the Jastrow-Slater as trial wave functions for Green's function [14] and diffusion [15] Monte Carlo. Since pairing becomes important at crossover a more general BCS wave function based on the Jastrow wave function was also employed. Extrapolating to a large number of particles they obtained $\beta(0)=-0.56 \pm 0.01$ and $\beta(0)=-0.58 \pm 0.01$ respectively in the unitary limit.

Recent measurements [4, 16, 17, 18, 19] of $\beta(0)$ confirm the unitarity limit near Feshbach resonances. Several experiments with trapped Fermi atoms have recently measured energies in the strongly interacting or dense limit near Feshbach resonances. The energy in the trap (excluding that from the harmonic oscillator potential) is $E / N=(3 / 8) E_{F} \sqrt{1+\beta(0)}$ where $E_{F}=(3 N)^{1 / 3} \hbar \omega$ is the Fermi energy in a trapped non-interacting gas. The first measurements by the Duke group [4] measured the energy of ${ }^{6} \mathrm{Li}$ Fermi atoms near a Feshbach resonance from expansion 
energies. These early measurements were later corrected for thermal energies and find $\beta(0)=-0.4 \pm 0.1$. [4, 17] With the discovery of a molecular BEC the Innsbruck group [19] has been able to measure the size of the atomic cloud, which scales with $(1+\beta)^{1 / 4}$, around the Feshbach resonance at very low temperatures, and find $\beta(0)=-0.68 \pm 0.1$. Recent accurate measurements find $\beta(0)=-0.49 \pm 0.02$. [20]

Other thermodynamic variables may in the unitarity limit depend on other universal parameters. For example, the number of closed channel molecules is proportional to $\beta^{\prime}(x)[32,33]$ and one obtains $\beta^{\prime}(0) \simeq-0.5$. From the slope of collective modes near the unitarity limit $\beta^{\prime}(0) \simeq-1.0$ [4, 17]. Generally, the universal function $\beta(x)$ is given by a Taylor expansion in terms of an infinite number number of derivatives $\beta^{(n)}(0)$, which all are universal parameters that determine all the universal thermodynamic functions.

Unlike neutrons, ultracold atoms can have many internal bound states and corresponding Feshbach resonances as the background magnetic field is increased. Thus solutions are multivalued for a given scattering length where the gas is in a metastable state. For example, starting from a non-interacting gas $(a=0)$ and increasing the scattering length $a>0$ we also approach an unitarity limit $a \rightarrow+\infty$ [21], which differs from that in the BCS-BEC crossover when the scattering length is decreased $a<0$. This unitarity limit is similar to that for bosons as $a \rightarrow+\infty$ [22], where the scattering length must be positive in order for the system to be stable. As will be described in detail the two-body wave function has a node and therefore the universal functions differs from those in the BCS-BEC crossover. In principle a new universal limit and crossover exist for each number of nodes, $n=1,2,3, \ldots$, in the two-body correlation function. These will, however, be increasingly unstable towards three-body losses. The $n=1$ has been observed for bosons in the atomicmolecular transition in a ${ }^{85} \mathrm{Rb} \mathrm{BEC}[23]$ and is in agreement with the predicted value from JS with $\kappa_{1}=2.80$. For fermions the predicted value is calculated below within the Jastrow-Slater approximations $\beta_{1}(0) \simeq 2.93$ which is compatible with a recent experiment [13]. This value is about six times $|\beta(0)|$ demonstrating that these two universal limits are very different.

\subsection{Thermodynamic crossover functions}

As argued above any thermodynamic function can only depend on one dimensionless scale such as $x$ at zero temperature when the range of interaction is sufficiently small. For example, the energy per particle in units of the Fermi energy is given in Eq. (1) in terms of the universal many-body crossover function $\beta(x)=E_{\text {int }} / E_{\text {kin }}$. It is well known in the dilute limit for Fermions [24]

$$
\frac{E}{N}=\frac{3}{5} E_{F}+\frac{\pi \hbar^{2}}{m} a n+\ldots
$$

to leading orders, whereas for molecular bosons 


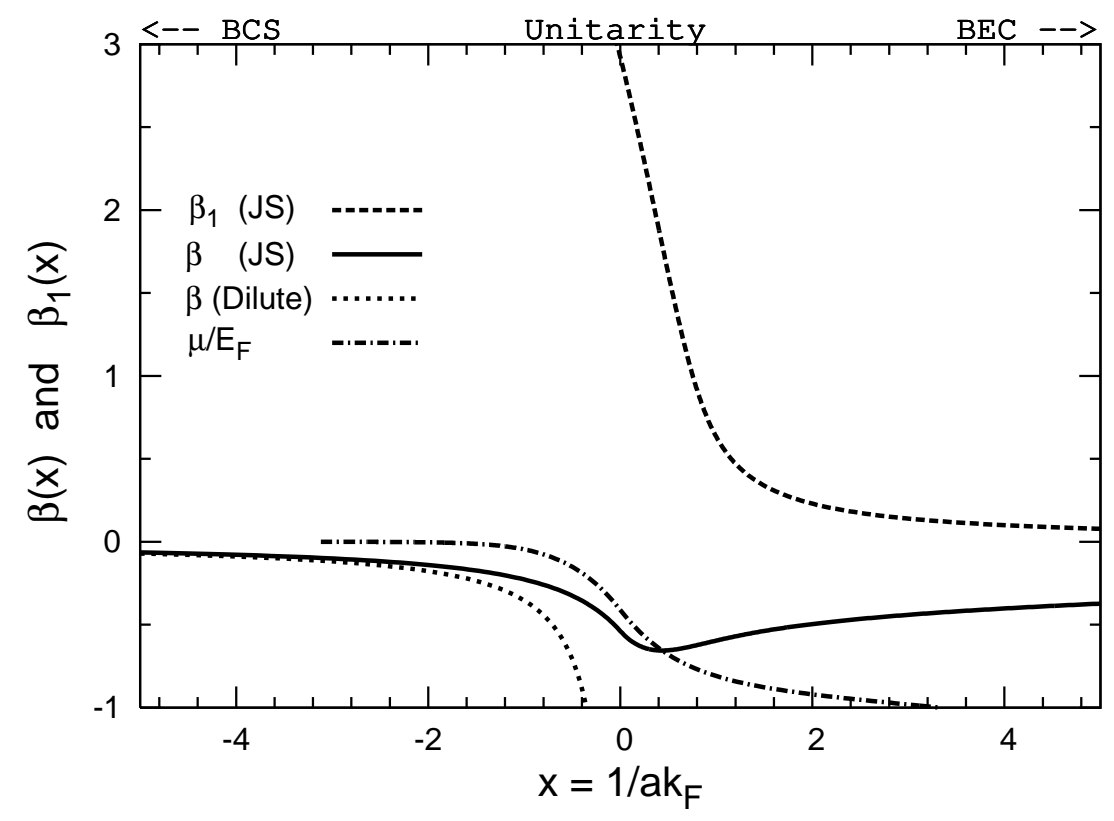

Fig. 2 The thermodynamic functions $\beta(x)$ and $\beta_{1}(x)$ within JS. Also shown are the BCS limit of Eq. (5) and the chemical potential in the Leggett crossover model (see text). On the BEC side $(x>0)$ the molecular energy been subtracted.

$$
\frac{E}{N}=-\frac{\hbar^{2}}{2 m a^{2}}+\frac{\pi \hbar^{2}}{2 m} a_{M} n+\ldots
$$

where the molecular scattering length is $a_{M} \simeq 0.6 a[54,16]$. Thus we obtain

$$
\beta(x)=\left\{\begin{array}{ll}
10 /(9 \pi x)+4(11-2 \ln 2) /\left(21 \pi^{2} x^{2}\right)+. . & , x \rightarrow-\infty \\
\beta(0)+\beta^{\prime}(0) x+. . & , x \simeq 0 \\
-5 x^{2} / 3-1+\left(a_{M} / a\right) 5 /(18 \pi x)+. . & , x \rightarrow+\infty
\end{array}\right\}
$$

In Fig. (2) various model calculations of the universal function $\beta(x)$ are shown.

The chemical potential is in terms of the universal function

$$
\mu(x)=\left(\frac{\partial E}{\partial N}\right)_{V, S}=E_{F}\left(1+\beta-\frac{1}{5} x \beta^{\prime}\right) .
$$

In both the hydrodynamic limit and for a superfluid gas the first sound is given by the adiabatic sound speed

$$
c_{S}^{2}(x)=\frac{n}{m}\left(\frac{\partial \mu}{\partial n}\right)_{V, S}=\frac{1}{3} v_{F}^{2}\left[1+\beta-\frac{3}{5} x \beta^{\prime}+\frac{1}{10} x^{2} \beta^{\prime \prime}\right],
$$

where $v_{F}=\hbar k_{F} / m$. 
The compressibility $\kappa=n^{-2}(\partial n / \partial \mu)$ is related to first sound as

$$
\kappa^{-1}=n^{2}\left(\frac{\partial \mu}{\partial n}\right)_{V, S}=m n c_{S}^{2}
$$

The pressure $P=n(\mu-E / N)=n^{2} d E / d V$ is

$$
P(x)=\frac{2}{5} E_{F} n\left[1+\beta-x \beta^{\prime} / 2\right] .
$$

The polytropic index $P \propto n^{\gamma+1}$ is defined as the logarithmic derivative of the pressure

$$
\gamma(x) \equiv \frac{n}{P} \frac{d P}{d n}-1=\frac{\frac{2}{3}(1+\beta)-x \beta^{\prime} / 2+x^{2} \beta^{\prime \prime} / 6}{1+\beta-x \beta^{\prime} / 2} .
$$

$\gamma$ approaches $2 / 3$ in both the dilute and unitarity limit whereas it approaches $\gamma=1$ in the molecular limit. This index determines the frequency of collective modes in traps [60].

We notice that all the above thermodynamic derivatives w.r.t. density only depend on one universal parameter $\beta(0)$ in the unitarity limit $x=0$ as argued above. Most thermodynamic quantities behave in the unitary gas as in a free Fermi gas except for the universal scaling factor $(1+\beta(0))$. This factor, however, cancels in the polytropic index and therefore $\gamma=2 / 3$ in both the unitarity and BCS limit and the collective modes are the same as has been verified experimentally [4].

\subsection{Finite temperature}

At finite temperature the thermodynamic functions also depend on the parameter $T / E_{F}$ (see, e.g., [3, 12, 25, 26]). Using $(\partial(E / N) / \partial T)_{V, N}=T(\partial s / \partial T)_{V, N}=c_{V}$, where $s=S / N$ is the entropy and $c_{V}$ the specific heat per particle, we obtain from Eq. (1)

$$
\beta\left(x, T / E_{F}\right)=\beta(x, 0)+\frac{5}{3} \int_{0}^{T} c_{V}\left(x, \frac{T^{\prime}}{E_{F}}\right) \frac{d T^{\prime}}{E_{F}} .
$$

The entropy is at temperatures well below the superfluid transition temperature $T \ll T_{c}$ given by phonon fluctuations

$$
s=\frac{2 \pi^{2}}{45 n}\left(\frac{T}{c_{S}}\right)^{3}
$$

Inserting in eq. 111) gives at low temperatures. 


$$
\beta\left(x, T / E_{F}\right)=\beta(x, 0)+5 \frac{T}{E_{F}} s .
$$

The temperature corrections scales as $\sim\left(T / T_{F}\right)^{4}$ and since $T \lesssim T_{c} \simeq 0.19 E_{F}$, the temperature dependence is almost flat as also observed in [20].

In a Fermi liquid $s=\left(\pi^{2} m^{*} / 2 m\right) T / E_{F}$, where $m^{*}$ is the effective mass, and thus

$$
\beta\left(x, T / E_{F}\right) \simeq \beta\left(x, T_{c} / E_{F}\right)+\frac{5 \pi^{2}}{6} \frac{m^{*}}{m} \frac{T^{2}-T_{c}^{2}}{E_{F}^{2}},
$$

when $T_{c}<T \ll E_{F}$. Recent accurate measurements find $\beta(0,0)=-0.49 \pm 0.01$ and $m^{*} / m=1.13 \pm 0.03[20]$ in the unitarity limit. These allow us to extract the Landau parameters at zero temperature $F_{0}^{s}=(1+\beta(0,0)) \mathrm{m}^{*} / \mathrm{m}-1 \simeq-0.42$ and $F_{1}^{s}=3\left(m^{*} / m-1\right) \simeq 0.39$. In the BCS limit $m^{*} / m=1+\left[8(7 \ln 2-1) / 15 \pi^{2}\right] a^{2} k_{F}^{2}$ and $F_{0}=1+(10 / 9 \pi) a k_{F}$.

As the temperature decreases towards the critical temperature the change in $\beta(T)$ from a Fermi liquid Eq. (14) towards a superfluid Eq. (13) allowed determination of $T_{c} / E_{F}=0.19 \pm 0.02[20]$.

At temperatures below $T<T_{c}$ the normal and superfluid components lead to two (first and second) sound modes in the collisional limit. In the BCS and BEC limit these are also referred to as the compressional and thermal sound modes. Their velocities $u_{1}$ and $u_{2}$ are given by the positive and negative solutions respectively of [27, 28]

$$
u^{2}=\frac{c_{S}^{2}+c_{2}^{2}}{2} \pm \sqrt{\left(\frac{c_{S}^{2}+c_{2}^{2}}{2}\right)^{2}-c_{T}^{2} c_{2}^{2}} .
$$

The thermodynamic quantities entering are the adiabatic $c_{S}^{2}=(\partial P / \partial n)_{S}$ and the isothermal $c_{T}^{2}=(\partial P / \partial n)_{T}$ compressional sound speed squared. The "thermal" sound wave $c_{2}^{2}=n_{s} s^{2} T / n_{n} c_{V}$ acts as a coupling or mixing term and is small in the two limits. Here, $n=n_{n}+n_{s}$ is the total, $n_{n}$ the normal and $n_{s}$ the superfluid density. The difference between the adiabatic and isothermal sound speed squared can also be expressed as

$$
c_{S}^{2}-c_{T}^{2}=\left(\frac{\partial s}{\partial n}\right)_{T}^{2} \frac{n^{2} T}{c_{V}}
$$

The mixing of the compressional and thermal sound modes is particular interesting at crossover where they mix and couple strongly. They undergo avoided crossing, i.e. the compressional change smoothly into a thermal sound mode and visa versa around the unitarity limit [25]. 


\subsection{Jastrow-Slater approximation}

The Jastrow and Jastrow-Slater (JS) approximation was among the earliest models applied to the unitarity limit and crossover [3]. It has the advantage that it provides analytical results for the universal parameters that are easy to understand and has proven to be quite accurate when compared to experiment. It gives an ansatz for the strongly correlated wavefunctions which is also the starting point as trial wavefunctions in Monte Carlo calculations [15]. Finally, JS can be generalized to describe both bosons and fermions with any number of spin states as well as other universal limits.

The Jastrow and the JS approximation methods were developed for strongly interacting and correlated Bose and Fermi fluids respectively such as ${ }^{4} \mathrm{He},{ }^{3} \mathrm{He}$ and nuclear matter in [29] and has more recently been applied to kaon condensation [30], cold atomic Fermi [21] and Bose [22] gases. As explained in these references the JS wave function

$$
\Psi_{J S}\left(\mathbf{r}_{1}, \ldots, \mathbf{r}_{N}\right)=\Phi_{S} \prod_{i, j^{\prime}} \phi\left(\mathbf{r}_{i}-\mathbf{r}_{j^{\prime}}\right)
$$

incorporates essential two-body correlations in the Jastrow function $\phi(r)$. The antisymmetric Slater wave function $\Phi_{S}$ for free fermions $\Phi_{S}$ insures that same spins are spatially anti-symmetric. The Jastrow wave function only applies to particles with different spins (indicated by the primes). The pair correlation function $\phi(r)$ can be determined variationally by minimizing the expectation value of the energy, $E / N=\langle\Psi|H| \Psi\rangle /\langle\Psi \mid \Psi\rangle$, which may be calculated by Monte Carlo methods that are fairly well approximated by including only two-body clusters. The basic idea of this method is that at short distances $r \ll r_{0}$ the Jastrow function $\phi(r)$ obeys the Schrödinger equation for a pair of particles interacting through a potential $U(r)$

$$
\left[-\frac{\hbar^{2}}{m} \frac{d^{2}}{d r^{2}}+U(r)\right] r \phi(r)=\varepsilon_{M} r \phi(r),
$$

where the eigenvalue energy of two atoms $\varepsilon_{M}=2 E_{\text {int }} / N$. Many-body effects become important, when $r$ is comparable to $r_{0}$, but are found to be small in lowest order constrained variation (LOCV [29, 3, 30, 21]). Here the boundary conditions that $\phi(r>d)$ is constant and $\phi^{\prime}(r=d)=0$ are imposed at the healing distance $d$, which is determined self consistently from number conservation (see [21] for details)

$$
\frac{v-1}{v} n \int_{0}^{d} \frac{\phi^{2}(r)}{\phi^{2}(d)} 4 \pi r^{2} d r=1
$$

Note that a given component only interact and correlate with the $(v-1)$ other components which explains the prefactor. In the dilute limit $\phi(r) \simeq 1$ and so $d=$ $(v /(v-1))^{1 / 3} r_{0}$. In the unitary limit $a \rightarrow \pm \infty$ the healing length now approaches $d=r_{0}(2 v /(v-1) 3)^{1 / 3}=(3 \pi /(v-1))^{1 / 3} k_{F}^{-1}$. Generally $d \simeq r_{0}$. The boundary 


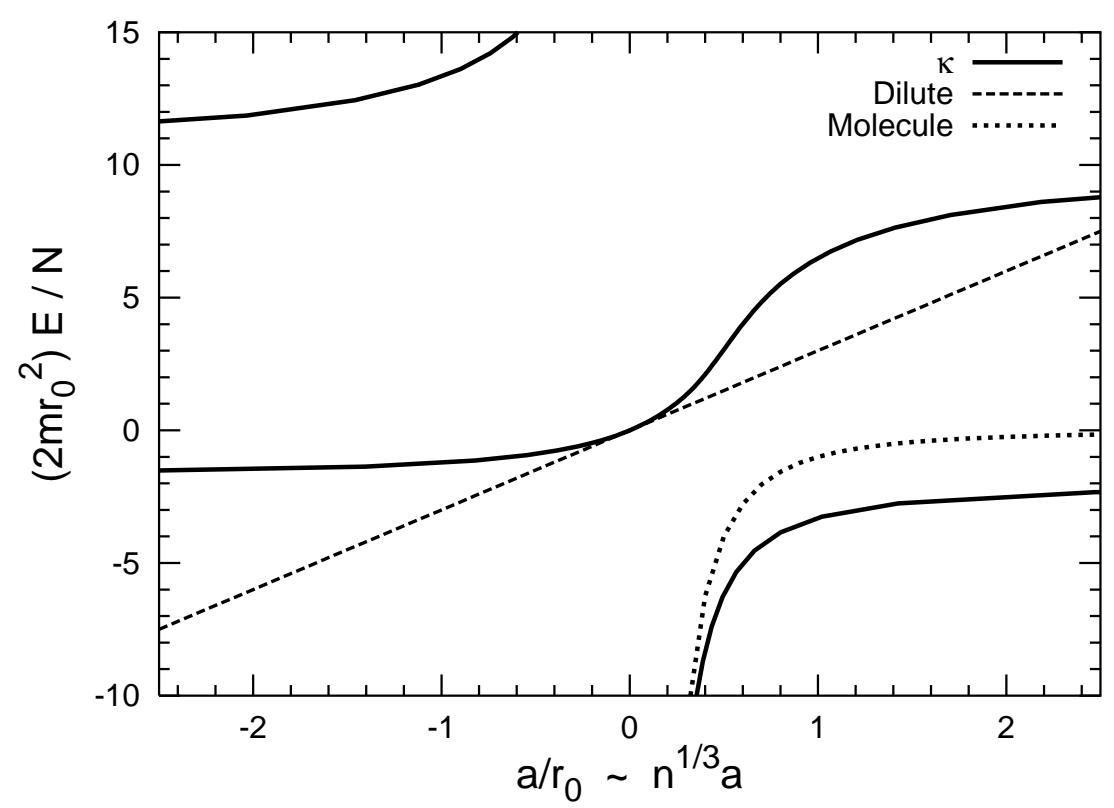

Fig. $3 \kappa^{2}$ vs. scattering length in the JS model in units of interparticle distance $r_{0}$.

condition at short distances is given by the scattering length $(r \phi)^{\prime} / r \phi=-1 / a$ at $r=0$.

\subsubsection{Attractive crossover}

For negative scattering lengths or negative energies $\varepsilon_{M}<0$ the solution to the Schrödinger equation gives a wave-function $r \phi(r) \propto \sinh [k(r-b)]$ for weak interactions which change to $r \phi(r) \propto \cosh [k(r-b)]$ ) for stronger interactions (see Fig. (44). The boundary conditions and number conservation determine the phase $k b$, the energy and the healing length $d$. For small scattering lengths $b=a$ whereas $b=0$ in the unitarity limit. The interaction energy $E_{\text {int }} / N=-\hbar^{2} k^{2} / 2 m$ is given by [31]

$$
\frac{a}{d}=\frac{\kappa^{-1} \tanh \kappa-1}{1-\kappa \tanh \kappa}
$$

with $\kappa=k d$. In the BCS limit Eq. (20) gives the correct interaction energy per particle, Eq. (3). In contrast the negative energy solution to Eq. (20) reduces in the unitarity limit to $\kappa \tanh \kappa=1$ with solution $\kappa_{0}=1.1997 \ldots$ As the scattering length cross over from $-\infty$ to $+\infty$ the negative energy state is analytically continued towards the molecular bound state with $E / N=-\hbar^{2} / 2 m a^{2}$ as $a \rightarrow+0$.

In addition to the interaction energy $E_{\text {int }}=\kappa^{2} / 2 m d^{2}$ as calculated above a kinetic energy $(3 / 5) E_{F}$ appears due to the Slater ground state. The total energy becomes 


$$
\frac{E}{N}=\frac{3}{5} E_{F}-\frac{\hbar^{2} \kappa^{2}}{2 m d^{2}} .
$$

From the definition Eq. (5) we obtain the universal function

$$
\beta=-\frac{5}{3}\left(\frac{\kappa}{d k_{F}}\right)^{2}
$$

In the unitarity limit $\kappa=\kappa_{0}=1.1997$ and $d k_{F}=(3 \pi /(v-1))^{1 / 3}$ and the universal parameter is for a general number of spin states

$$
\beta(0)=-\frac{5}{3}\left(\frac{v-1}{3 \pi}\right)^{2 / 3} \kappa_{0}^{2} .
$$

Thus in a two-component system $\beta(0)=-0.54$ which lies between Monte Carlo results $\beta(0) \simeq-0.56[15]$ and recent experimental data $\beta(0) \simeq-0.49$ [20].

The universal function $\beta(x)$ is shown in Fig. (2) for the JS approximation. Also shown is the chemical potential within the Leggett crossover model which will be discussed below in connection with pairing. The JS model includes self energies and is therefore a better approximation on the BCS side $(x<0)$. On the BEC side $(x>0)$ both models approach the molecular energy (subtracted in Fig. (2)) but to next orders both model overestimates the energy: the JS model by the Slater energy and the Leggett model by overestimating the molecular scattering length $a_{M}$ by a factor $\sim 3$.

The dependence on the scattering length is given by Eq. 20). By taking the derivative on both sides w.r.t. $x$ we obtain

$$
\beta^{\prime}(0)=-\frac{10}{3} \frac{1-\kappa_{0}^{-2}}{(3 \pi)^{1 / 3}} \simeq-0.48 .
$$

This JS prediction is somewhat lower than that of Monte Carlo: $\beta^{\prime}(0)=-1.0 \pm 0.1$ [14, 15]. The slopes of the axial and longitudinal collective frequencies of trapped unitary Fermi gases are directly proportional to $\beta^{\prime}(0)$ and measurements also give $\beta^{\prime}(0) \simeq-1.0$ [4, 17]. Analysis of the number of closed channel molecules indicates $\beta^{\prime}(0) \simeq-0.5\left[33\right.$, 32]. The Leggett pairing model $\beta^{\prime}(0) \simeq-1.0$. (2.5)

The universal parameter $\beta^{\prime}(0)$ will be related to short range correlations in sec.

\subsubsection{Repulsive crossover}

Starting from a positive scattering length the and positive interaction energy the solution to the Schrödinger equation gives a wave-function $r \phi(r) \propto \sin [k(r-b)]$ (see Fig. (4)). The boundary conditions and number conservation gives [22] 


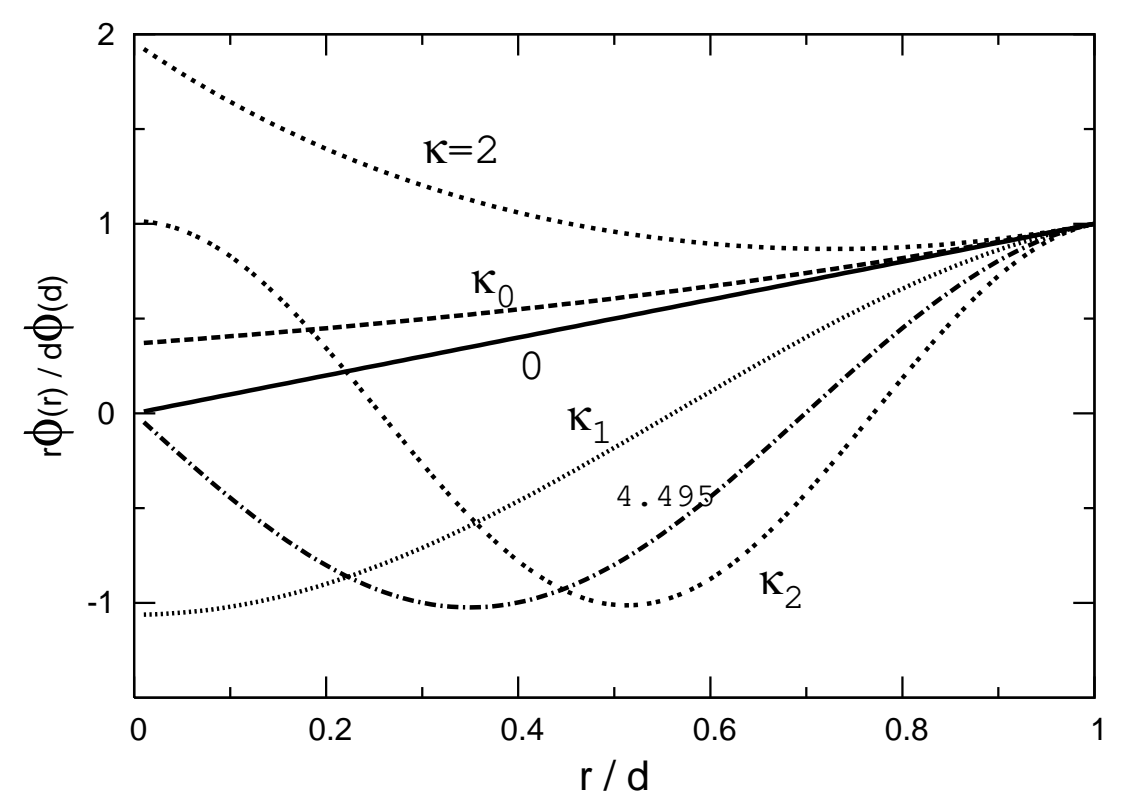

Fig. 4 The JS wave function $r \phi(r) / d \phi(d)$ in the dilute limit $(\kappa=0)$, the attractive unitarity limit $\left(\kappa_{0}\right)$, towards the mBEC $(\kappa=2)$, the repulsive unitarity limit with one $\left(\kappa_{1}\right)$ and two nodes $\left(\kappa_{2}\right)$.

$$
\frac{a}{d}=\frac{\kappa^{-1} \tan \kappa-1}{1+\kappa \tan \kappa} .
$$

As in the BCS limit Eq. 25) gives the correct interaction energy per particle in the dilute limit, Eq. (3). In the unitarity limit $a \rightarrow+\infty$, the positive energy solution reduces to $\kappa \tan \kappa=-1$ with multiple solutions $\kappa_{1}=2.798386$.., $\kappa_{2}=6.1212$.., etc., and asymptotically $\kappa_{n}=n \pi$ for integer $n$. Generally, $n=0,1,2, \ldots$ is the number of nodes in the JS wavefunction and each determines a new universal limit with universal parameters depending on the number of nodes. The phase in the wave function is $k b=\pi(n-1 / 2)$ whenever the unitarity limit of $n$ nodes is encountered.

The resulting energy is

$$
\frac{E}{N}=\frac{3}{5} E_{F}+\frac{\hbar^{2} \kappa^{2}}{2 m d^{2}}
$$

and we obtain from Eq. (1)

$$
\beta(x)=\frac{5}{3}\left(\frac{\kappa}{d k_{F}}\right)^{2}
$$

Because Eq. (25) have a string of solutions for given $a, \kappa$ and $\beta$ are multivalued functions for a given scattering length or $x$, which we distinguish by an index $\beta_{n}$ referring to the number of nodes $n=0,1,2, \ldots$. The attractive case discussed above 
is the $n=0$ case, i.e. universal function in the BCS-BEC crossover is also $\beta=\beta_{0}$. $n=1$ is the first weakly repulsive crossover where a ferromagnetic phase transition may occur and is also shown in Figs. (2) and (3).

The universal parameter in this repulsive unitarity limit is

$$
\beta_{1}(x=0)=+\frac{5}{3}\left(\frac{v-1}{3 \pi}\right)^{2 / 3} \kappa_{1}^{2} \simeq 2.93(v-1)^{2 / 3} .
$$

The index denotes that this universal parameter is for the wave function with one node. It has recently been measured in a ${ }^{6} \mathrm{Li}$ gas in two spin states [13]. The chemical potential in the optical trap almost doubles going from the non-interacting to the unitarity limit. As shown below it scales as $\mu \propto \sqrt{1+\beta_{1}(0)}$ giving $\beta_{1}(0) \sim 3$ compatible with JS. However, the experiments also indicate a phase transition in repulsive unitarity limit from a paramagnetic to a ferromagnetic phase. This phase transition is supported by the JS models as will be shown in sec. (2.7).

As for the attractive crossover we calculate within JS the derivative from Eq. 25)

$$
\beta_{1}^{\prime}(0)=-\frac{10}{3} \frac{\left(1-\kappa_{1}^{-2}\right)}{(3 \pi)^{1 / 3}} \simeq-1.38
$$

\subsection{Short range correlations}

The short range two-body correlations are connected to thermodynamic quantities of the system [34]. We shall here derive one important example at zero temperature and use the Jastrow-Slater results above to actually calculate a new universal number. We refer to [35, 26] for finite temperature extensions.

Following [26] we scale the two-body potential by a factor $\lambda$ and obtain from Feynmann-Hellmann

$$
\frac{\partial E / V}{\partial \lambda}=\frac{1}{V}\left\langle\frac{\partial H}{\partial \lambda}\right\rangle=\int d^{3} r U(r)\left\langle\psi_{1}^{\dagger}(\mathbf{r}) \psi_{2}^{\dagger}(0) \psi_{2}(0) \psi_{1}(\mathbf{r})\right\rangle_{\lambda}
$$

Here the correlation function at short ranges is

$$
\left\langle\psi_{1}^{\dagger}(\mathbf{r}) \psi_{2}^{\dagger}(0) \psi_{2}(0) \psi_{1}(\mathbf{r})\right\rangle_{\lambda}=\phi_{\lambda}^{2}(r)=C\left(\frac{1}{r}-\frac{1}{a_{\lambda}}\right)^{2},
$$

where $\phi_{\lambda}$ is the solution to the two-body Schrödinger equation (18) but with potential $\lambda U(r)$. $C$ is a normalization factor such that $(1 / V) \int d^{3} r \phi_{\lambda}^{2}(r)=(n / 2)^{2}$, and will later be interpreted as the correlation strength.

Taking the derivative of the Schrödinger equation w.r.t. $\lambda$, multiplying by $r \phi_{\lambda}$, and integrating over $r$ we obtain

$$
\int_{0}^{r_{c}} d r U(r)\left(r \phi_{\lambda}(r)\right)^{2}=-\frac{C}{m} \frac{\partial a^{-1}}{\partial \lambda},
$$


where $r_{c}$ is any range between $R \ll r_{c} \ll r_{0}$. Combining Eqs. (30) and (32) gives

$$
\frac{\partial E / V}{\partial a^{-1}}=-\frac{4 \pi C}{m} .
$$

From Eq. (1) we can relate the correlation strength to the derivative of the universal functions $\beta_{n}(x)$ as

$$
C_{n}=-\frac{k_{F}^{4}}{40 \pi^{3}} \beta_{n}^{\prime}(x)
$$

where the node index $n$ distinguishes the various universal functions as discussed above. In the unitarity limits we can determine $C_{n}(x=0)$ by inserting $\beta_{0}^{\prime}(0)=-0.54$ or $\beta_{1}^{\prime}(0)=-1.38$ from JS Eqs. (24) and (29), etc. $C_{n}(x)$ can also be obtained directly from the JS wave-function, normalization and Eq. 31).

The Fourier transform of the wave-function at large momenta is determined by the short range correlations $\phi(k)=\int d^{3} r e^{i \mathbf{k} \cdot \mathbf{r}} \phi(r) \simeq 4 \pi \sqrt{C} / k^{2}$. Therefore the momentum distribution for a spin state has the tail

$$
n_{\sigma}(k)=\phi^{2}(k)=(4 \pi)^{2} C / k^{4},
$$

at large momenta as is well known for Fermi liquids [24]. The correlation strength is directly measured in the number of closed channel molecules [32]

$$
N_{M}=\frac{\partial E}{\partial a^{-1}} \frac{\partial a^{-1}}{\partial B} \frac{1}{\mu_{M}}
$$

where $\mu_{M}$ is twice the magnetic moment of the atom. The scattering length depends on the applied magnetic field as $a \simeq a_{b g}\left[1-\Delta B /\left(B-B_{0}\right)\right]$ near a Feshbach resonance at $B_{0}$. Experiments find for the BCS-BEC crossover [32] $\beta_{0}^{\prime}(0) \simeq-0.5$ which agrees well with that of JS and is compatible with model estimates [33].

\subsection{Instability in multicomponent systems}

The original Bersch problem was intended for two-component systems as neutron matter or spin-balanced ultracold atoms in two hyperfine states. Early extrapolations of the dilute energy of Eq. (3) to $a \rightarrow-\infty$ led to the belief that the unitarity limit was unstable towards collapse [36] as a nuclear gas whereas in fact the two-component system is the only stable unitary gas. The predicted scaling for the neutron gas at subnuclear density as given by the unitary gas of Eq. (1) triggered the memory of Vijay Pandharipande[37], who had the impression that Bethe and Brueckner were aware of this scaling when they looked at low density neutron matter in neutron 
star context back in the '50s [38, 29] 1] Calculations by Carlson et al. [14] have later confirmed this scaling in neutron gases.

Multicomponent systems are now studied, e.g. ${ }^{6} \mathrm{Li}$ with three hyperfine states [5], ${ }^{137} \mathrm{Yb}$ with six nuclear spin states [6], and heteronuclear mixtures of ${ }^{40} \mathrm{~K}$ and ${ }^{6} \mathrm{Li}$ [7]. Such multi-component systems have intriguing similarities with neutron, nuclear and quark matter where color superconductivity between the 2 spin, 8 color and 2-3 flavor states may occur [8].

For the gas to be stable towards collapse in the unitarity limit the energy must be positive, i.e., $1+\beta(0)>0$. In the JS approximation we obtain in the unitary limit from Eq. (23) $\beta(0)=0,-0.54,-0.85,-1.12, \ldots$, for $v=1,2,3,4, \ldots$ spin states respectively. Therefore the JS approximation predicts that up to $v \leq 3$ spin states are stable in the unitary limit whereas in the Galitskii approximation only $v=1,2$ are stable [3]. Pauli blocking is effectively reduced in many component system and can only stabilize one, two and perhaps three component Fermi systems in the unitary limit. The stability of two spin states towards collapse has been confirmed for a ${ }^{6} \mathrm{Li}$ and ${ }^{40} \mathrm{~K}$ gases near Feshbach resonances. The marginal case $v=3$ has been studied with ${ }^{6} \mathrm{Li}$ atoms, which have three spin states with broad and close lying Feshbach resonances, and although the loss rate of atoms is large near Feshbach and Efimov resonances [5] the gas is sufficiently long lived for measurements and does not collapse. It has long been known that neutron star matter [39] with two spin states likewise has positive energy at all densities whereas for symmetric nuclear matter with two spin and two isospin states, i.e. $v=4$, the energy per particle is negative. Nuclear matter is therefore unstable towards collapse and subsequent implosion, spinodal decomposition and fragmentation at subnuclear densities [40]. Above nuclear saturation densities, $k_{F} R \gtrsim 1$, short range repulsion stabilizes matter up to maximum masses of neutron stars $\sim 2.2 M_{\odot}$, where gravitation makes such heavy neutron stars unstable towards collapse [39]. The conjecture is therefore that the $v>3$ Fermi systems are unstable and non-universal in the unitarity limit $a \rightarrow-\infty$. Bose atoms corresponds to $v=\infty$, since no Pauli blocking applies, and are unstable for negative scattering lengths.

The $v=3$ system is relevant for several reasons. Traps with ultracold ${ }^{6} \mathrm{Li}$ atoms with three hyperfine states are sufficiently stable and long lived [5] to be studied in detail. The three Feshbach resonances are, however, separate in magnetic field so that the unitarity limit is not simultaneous for the three components. The three body system has interesting Efimov states which are non-universal [41, 42], i.e. besides the scattering length the system depends on an additional potential parameter such as the effective range. This non-universality persists for three bosons confined in a trap [43] and non-universality is therefore expected for a gas with three state Fermi atoms as experiments for the ${ }^{6} \mathrm{Li}$ system also indicates [5]. Therefore three component systems do not have an universal limit and are only marginally stable, i.e. stable towards collapse but suffer three-body losses.

In spin polarized systems or systems with different densities of the spin components the stability conditions depends on the various component densities. Also the

${ }^{1}$ However, no written reference has been found in the works of Bethe, Brueckner or Pandharipande so far. 
system may undergo phase separation into a more symmetric phase and an asymmetric phase as, e.g., nuclei and a neutron gas in the inner crust of neutron stars. Similarly, spin polarized atoms at low temperature in traps may for strong attractive interactions separate into a paired spin balanced phase in the centre with a mantle of excess spin atoms. Likewise phase separation of strongly repulsive fermions may separate into domains of ferromagnetic phases in the centre with a paramagnetic mantle around.

\subsection{Repulsive interactions and itinerant Ferromagnetism}

For a small positive scattering length the Fermi gas is a paramagnet (PM). For stronger repulsion Stoner [44] predicted a phase transition to a ferromagnet (FM) which a recent experiment claim to have observed [13]. Stoner's argument was based on the dilute equation of state of Eq. (3) which generally for a spin polarized two-component system of total density $n=n_{\downarrow}+n_{\uparrow}$ and polarization $\eta=\left(n_{\downarrow}-n_{\uparrow}\right) / n$ is

$$
E / N=\frac{3}{10} E_{F}\left[(1+\eta)^{5 / 3}+(1-\eta)^{5 / 3}+\frac{20}{9 \pi}(1+\eta)(1-\eta) k_{F} a\right] .
$$

Expanding for small polarization gives an equation of the Ginzburg-Landau type

$$
E / N E_{F} \simeq \frac{3}{5}+\frac{2}{3 \pi} a k_{F}+\frac{1}{3}\left(1-\frac{2}{\pi} a k_{F}\right) \eta^{2}+3^{-4} \eta^{4}+\mathscr{O}\left(\eta^{6}\right) .
$$

It predicts a second order phase transition at $a k_{F}=\pi / 2$ from a PM to a FM with polarization $\eta= \pm \sqrt{27\left(2 a k_{F}-1 / \pi\right)}$. Due to the small fourth order coefficient it quickly leads to a locally fully polarized system $\eta= \pm 1$.

Unfortunately the predicted transition occurs close to the unitarity limit where the dilute equation of state is not valid. Higher orders may be important as exemplified by including the next order correction of order $a^{2}$. It changes the transition from second to first order [45] at low temperatures up to a tri-critical point at $T_{c} \simeq 0.2 T_{F}$, where the transition becomes second order again. However, the dilute expansion remains invalid in the unitarity limit.

The Jastrow-Slater approximation extends to the unitarity limit also for positive scattering lengths as discussed above. Number conservation of the various spin densities is automatically included in the healing length, see e.g. Eq. (19). As result the energy is

$$
\begin{aligned}
\frac{E}{N}= & \frac{3}{10} E_{F}\left[(1+\eta)^{5 / 3}+(1-\eta)^{5 / 3}+\beta_{1}(1+\eta)(1-\eta)^{2 / 3}+\beta_{1}(1+\eta)^{2 / 3}(1-\eta)\right] \\
& +\mathscr{O}\left(\eta^{6}\right) .
\end{aligned}
$$


$\beta_{1}(x, \eta)$ is now also a function of polarization but for simplicity we shall ignore the dependency this dependency as we expect it to be minor. Expanding for small polarization we find

$$
E / N E_{F} \simeq \frac{3}{5}\left(1+\beta_{1}\right)+\frac{1}{3}\left(1-\frac{7}{5} \beta_{1}\right) \eta^{2}+3^{-4}\left(1-\beta_{1}\right) \eta^{4} .
$$

Truncating to order $\eta^{4}$ would (erroneously) predict a second order phase transition from a PM to a FM at $\beta_{1}(x)=5 / 7 \simeq 0.71$. The fourth order term is even smaller than the dilute prediction of Eq. (38), and it is therefore necessary to include higher orders. By equating the energy of the unpolarized gas, $\sim\left(1+\beta_{1}\right)$ with that of a fully polarized gas, $\sim 2^{2 / 3}$, we find a first order transition at $\beta_{1}=2^{2 / 3}-1 \simeq 0.59$, since this value is smaller than $5 / 7$. In view of the approximations made in this model calculation and the proximity of the two $\beta_{1}$ values for the first and second order transitions, we can not reliably determine whether the order of the PM-FM transition is first or second. However, since the repulsive interaction energy in the unitarity limit $\beta_{1}(0) \simeq \kappa_{1}^{2} /(3 \pi)^{2 / 3} \simeq 2.93$. is much larger than the critical value $\beta_{1} \simeq 0.59$ we can safely conclude that the transition to a FM does take place at a value corresponding to $a k_{F} \simeq 0.85$.

In a recent experiment the transition is observed around $a k_{F}^{0} \simeq 2.2$ at temperatures $T / T_{F}=0.12$ and $a k_{F}^{0} \simeq 4.2$ at $T / T_{F}=0.22$ [13]. Presumably, the critical value for $a k_{F}^{0}$ is smaller at zero temperature. Also the Fermi wavenumber $k_{F}^{0}$ is the central value for a non-interacting gas which is larger than the average value over the trap of the gas that is further expanded due to repulsive interactions. More experiments will determine $\beta_{1}(x)$, a possible ferromagnetic phase and critical value for $a k_{F}$.

When the number of atoms in the two spin states is balanced the ferromagnetic domains of $\eta= \pm 1$ coexist. Their domain sizes may be to small to observe in present experiments [13]. The densities of the two components will, however, have interesting distributions for unbalanced two-component systems in traps, where the minority component will be suppressed in the centre and both phase separation and ferromagnetism can occur. In three component systems, when there are more than one Feshbach resonance as in ${ }^{6} \mathrm{Li}$, with Feshbach magnetic field such that two resonances $a_{12}$ and $a_{13}$ are large but $a_{23}$ small, the atoms will separate between a FM phase of 1 and a mixed FM phase of $2+3$ with different densities.

It should be emphasized that for positive scattering lengths the wave function and thus the correlations function between fermions of unlike spin and bosons $\chi=$ $r \phi \sim \sin (k r-b)$ has a node somewhere within the interparticle distance $\left[0 ; r_{0}\right]$ (see [22] and Fig. (4)). It does not vanish as $r \rightarrow 0$ as does the wave function for a short range repulsive potential as in hard sphere scattering, where $a \simeq R$. Therefore the Gutzwiller approximation discussed in [46] applies to hard sphere gases, strongly correlated nuclear fluids and liquid helium but not to the repulsive unitarity limit of ultracold gases (see Fig. (1). 


\section{Pairing in uniform systems and the BCS-BEC Crossover}

Experiments on superfluid Fermi gases have recenty confirmed the BCS to BEC crossover models [9, 10, 11, 56, 57] for pairing that was developed after the invention of BCS theory. The development of BCS-BEC crossover will be described historically with increasing level of complexity.

\subsection{BCS limit}

Bardeen, Cooper and Schriffer (BCS) first wrote down the famous gap equation 47] for an attractive two-body interaction $U(r)<0$

$$
\Delta_{\mathbf{p}}=-\frac{1}{V} \sum_{\mathbf{p}^{\prime}} U\left(\mathbf{p}^{\prime}, \mathbf{p}\right) \frac{1-2 f\left(E_{\mathbf{p}^{\prime}}\right)}{2 E_{\mathbf{p}^{\prime}}} \Delta_{\mathbf{p}^{\prime}}
$$

which can be elegantly derived via the Bogoliubov transformation [48]. Here, $f(\varepsilon)=$ $(\exp (\varepsilon-\mu) / T)+1)^{-1}$ is the Fermi distribution function, $E_{\mathbf{k}}=\sqrt{\left(\varepsilon_{\mathbf{k}}-\mu\right)^{2}+\Delta_{0}^{2}}$ the quasi-particle energy and $\varepsilon_{\mathbf{k}}=\hbar^{2} k^{2} / 2 m$ the free particle energy. In the BCS limit number conservation insures that $\mu=E_{F} . U\left(\mathbf{p}, \mathbf{p}^{\prime}\right)=U\left(\mathbf{p}-\mathbf{p}^{\prime}\right)$ is the Fourier transform of $U(\mathbf{r})$. In metals phonons provide a small residual attractive interaction $U\left(\mathbf{p}^{\prime}, \mathbf{p}\right) \simeq-V_{p h}$ with a cutoff of order the Debye frequency $\omega_{D}$. As result we obtain from Eq. (41) the BCS s-wave gap $\Delta_{0}=2 \hbar \omega_{D} e^{-1 / N(0) V_{p h}}$ at zero temperature.

In the years immediately after BCS was developed the gap equation was generalized in terms of scattering lengths[49]. When the interaction $U(\mathbf{r})$ is short range, its Fourier transform $U\left(\mathbf{p}-\mathbf{p}^{\prime}\right)$ is long range in momentum. It is then convenient to replace the interaction by its scattering matrix $T=U+U G_{0} T$, where $G_{0}=1 /\left(2 \varepsilon_{\mathbf{k}}-i \delta\right)$, is the vacuum propagator for two particles. At low momenta the scattering matrix is given by the s-wave scattering length $T=4 \pi \hbar^{2} a / m \equiv U_{0}$. Eliminating $U$ in the gap equation (41) gives

$$
1=\frac{U_{0}}{2 V} \sum_{\mathbf{k}}\left[\frac{1}{\varepsilon_{\mathbf{k}}}-\frac{1-2 f\left(E_{\mathbf{k}}\right)}{E_{\mathbf{k}}}\right] .
$$

Note that the difference between the vacuum and in medium Green's functions automatically cuts off the high momenta, which are now included in the scattering length. Solving this gap equation at zero temperature for a Fermi gas interacting through an attractive scattering length $a<0$ gave a pairing gap in the dilute limit, $\left.|a| k_{F} \ll 1,49\right]$

$$
\Delta_{0}=\frac{8}{e^{2}} E_{F} \exp \left[\frac{\pi}{2 a k_{F}}\right] .
$$




\subsection{Induced Interactions}

Gorkov 2 pointed out that many-body effects (induced interactions) lead to the next order correction in the interaction [50, 51, 52, 53]

$$
\begin{aligned}
U_{\text {ind }}\left(\mathbf{p}^{\prime}, \mathbf{p}\right) & =-\frac{U_{12}^{2}}{M} \sum_{\mathbf{q}} \frac{f\left(\xi_{1}\left(\mathbf{k}^{\prime}+\mathbf{q}\right)\right)-f\left(\xi_{1}(\mathbf{q})\right)}{\xi_{1}\left(\mathbf{k}^{\prime}+\mathbf{q}\right)-\xi_{1}(\mathbf{q})} \\
& +\sum_{j, \mathbf{q}} \frac{U_{1 j} U_{2 j}}{M} \frac{f\left(\xi_{3}(\mathbf{k}+\mathbf{q})\right)-f\left(\xi_{3}(\mathbf{q})\right)}{\xi_{3}(\mathbf{k}+\mathbf{q})-\xi_{3}(\mathbf{q})} .
\end{aligned}
$$

$f$ is the Fermi distribution of $\xi_{j}(\mathbf{q})=\varepsilon_{\mathbf{q}}-\mu_{j}, \mathbf{k}^{\prime}=\mathbf{p}+\mathbf{p}^{\prime}$ and $\mathbf{k}=\mathbf{p}-\mathbf{p}^{\prime}$, where $\mathbf{p}_{i}$ are the momenta of the two pairing spin states. Induced interactions due to particlehole loop diagrams from $j=3, \ldots, v$ spin states are responsible for the second sum in Eq. (44) and has opposite sign. For two components, however, it is absent.

In the two-component spin-balanced system the induced interactions effectively leads to a second order correction to the scattering length $a \rightarrow a+(2 / 3 \pi) \ln (4 e) k_{F} a^{2}$ [50, 51], where $a=a_{12}$ and $U_{12}=U_{0}=4 \pi \hbar^{2} a / m$. Including this correction in Eq. (43) reduces the gap by a factor $(4 e)^{1 / 3} \simeq 2.2$

$$
\Delta=\left(\frac{2}{e}\right)^{7 / 3} E_{F} \exp \left[\frac{\pi}{2 a k_{F}}\right] .
$$

The induced interactions consists of a repulsive direct part and an attractive part due a loop diagram which is therefore proportional to the number of components $v$. The induced interactions therefore scale as $(3-v)$ in a system of $v$ spin balanced multi-components with the same scattering length. The corresponding gap is

$$
\Delta=(4 e)^{v / 3-1} \Delta_{0} .
$$

For spin polarized systems or for the ${ }^{6} \mathrm{Li}$ systems with three different scattering lengths the pairing gap is described in Refs. [52]. Adding bosons enhance pairing [51].

In the unitary limit $k_{F}|a| \gtrsim 1$ the gap is of order the Fermi energy [10]. Extrapolating (45] to $a k_{F} \rightarrow \pm \infty$ [3] gives a number $\Delta=0.49 E_{F}$ close to that found from odd-even staggering binding energies $\Delta=0.54 E_{F}$ calculated by Monte Carlo [14]. The crossover model of Leggett described below gives a somewhat larger gap $\Delta^{\text {Leggett }} / E_{F}=0.69$. Such values of order the Fermi energy are one or two orders of magnitude larger those found in metals and high temperature superconductivity and was met by disbelief among condensed matter physicist and the crossover was considered academic. Only after the realization and confirmation of the BCS-BEC crossover in experiments with ultracold atoms was its significance acknowledged.

\footnotetext{
${ }^{2}$ Inquiring into details about their calculation some 40 years later Gorkov only remembered “...that it was a particular difficult calculation!"
} 


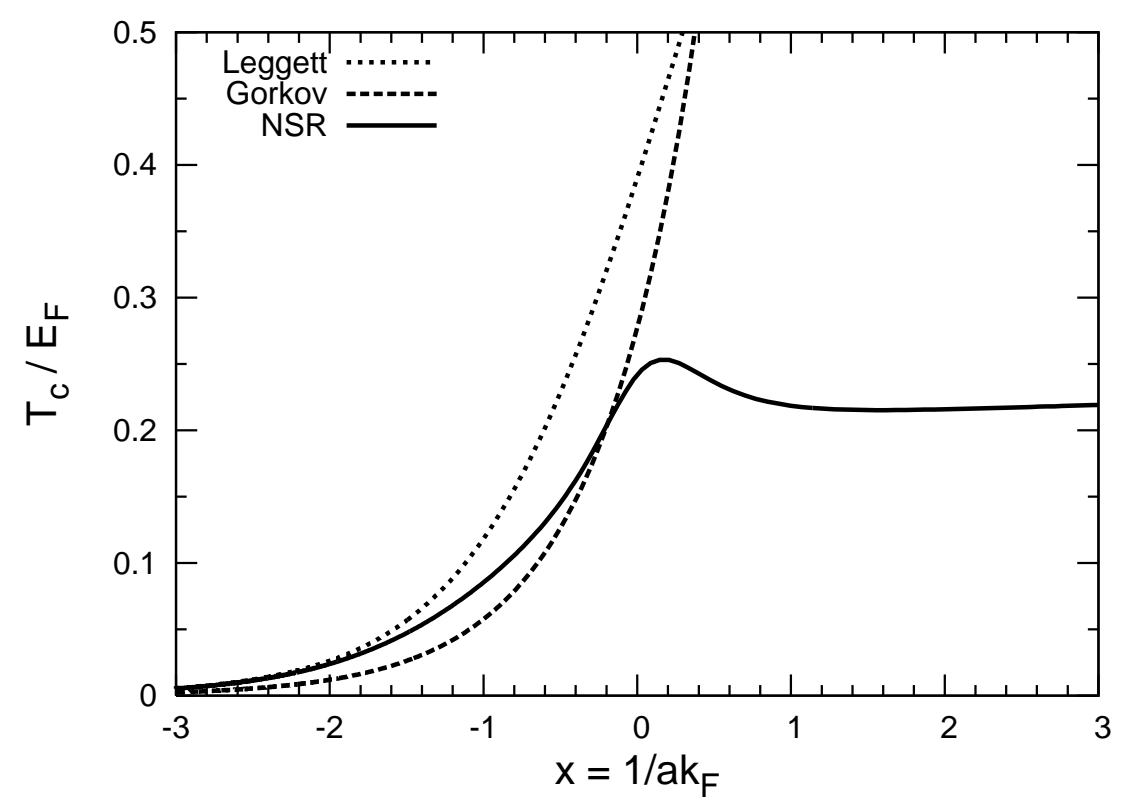

Fig. 5 Superfluid transition temperatures from the Leggett crossover, Gorkov and NSR.

It also follows from the BCS gap equation that the gap and thus superfluidity and superconductivity all vanish at a critical temperature

$$
T_{c}=\frac{e^{C_{E}}}{\pi} \Delta \simeq 0.567 \Delta,
$$

for any weak interaction ( $C_{E}=0.577 \ldots$ is Euler's constant). In the unitarity and BEC limits the critical temperature is no longer proportional to the gap.

\subsection{Leggett's BCS-BEC Crossover}

Eagles and Leggett made the important step of connecting BCS pairing to a BEC of molecular bosons via crossover. Eagles gave an early treatment of the crossover from BCS superconductivity to a BEC in the context of systems with low carrier concentrations [9]. For pedagogical reasons we will follow Leggett who solved the gap equation of Eq. (42) and number conservation

$$
N=\sum_{\mathbf{k}}\left[1-\frac{\varepsilon_{\mathbf{k}}-\mu}{E_{\mathbf{k}}}\right],
$$


self consistently in the crossover. The beauty of Leggett's crossover model Eqs. (42) and (48) is that it describes the crossover continuously as function of the parameter $x=1 / a k_{F}$ from the BCS limit $(x \rightarrow-\infty)$ over the unitarity limit $(x=0)$ to the BEC limit $(x \rightarrow+\infty)$. It is important to include (pairing) interactions in the chemical potential through number conservation as it changes the chemical potential dramatically.

In the dilute (BCS) limit the gap equation leads to the standard BCS gap of Eq. (43) - not including the Gorkov correction. The chemical potential is $\mu=E_{F}$ and does not include the standard mean field Hartree-Fock correction of a dilute gas.

In the BEC limit the pairing gap approaches

$$
\Delta=4 E_{F} / \sqrt{3 \pi a k_{F}} .
$$

The chemical potential approaches half of the molecular binding energy $\varepsilon_{M}=$ $-\hbar^{2} / m a^{2}$,

$$
\mu=-\frac{\hbar^{2}}{2 m a^{2}}+\frac{\pi \hbar^{2} n a}{m},
$$

plus the BEC mean field corresponding to a molecular scattering length of $a_{M}=2 a$. Four-body [54], Monte Carlo calculations [15] and experiments [16] do, however, indicate that $a_{M} \simeq 0.6 a$

On the BCS side the minimum quasiparticle energy is $\Delta$ and occur when $k=k_{F}$. On the BEC side the chemical potential is negative and the minimum quasiparticle excitation energy is the quasiparticle energy for $\mathbf{k}=0$

$$
E_{q p}=\sqrt{\mu^{2}+\Delta^{2}} .
$$

The quasiparticle energy is observed in the spin excitation response function [76, 57.

In the Leggett model the gap vanishes in the BEC limit at a critical temperature $T_{c}^{\text {dissoc }}=\left|\varepsilon_{M}\right| / \ln \left(\left|\varepsilon_{M}\right| / E_{F}\right)^{3 / 2}$, which is smaller than the quasi-particle excitation energy. It is not the on-set temperature for superfluidity but rather a molecular pair dissociation temperature [11]. The onset of superfluidity occurs at a lower critical temperature for a molecular BEC, $T_{c}^{B E C}$.

The Leggett model fails in the BEC limit because of the basic assumption that only opposite momenta fermions (zero total momentum pairs) can pair. Pairs with non-zero momenta are thermal excitations of molecular bosons and including such degrees of freedom lowers the critical temperatures increasingly towards the (molecular) BEC limit. 


\subsection{NSR}

The model of Noziéres \& Schmit-Rink (NSR) [11] extends the Leggett model so that it correctly describes the critical temperature in the molecular BEC limit. We will therefore give a brief outline of NSR with emphasis on how pair motion is included and how it corrects the Leggett model in the BEC limit. Also, the NSR approach is formulated such that it applies to optical lattices discussed in sec. (5) with few but crucial differences.

In the NSR model the (molecular) pair momentum $\mathbf{q}$ is included in the twoparticle correlation function. To lowest order it is given by the propagator for two free atoms

$$
\begin{aligned}
\Pi\left(\mathbf{q}, \omega_{v}\right) & =\frac{T}{V} \sum_{\mathbf{k}, \omega_{m}} G_{0}\left(\mathbf{k}, i \omega_{m}\right) G_{0}\left(\mathbf{q}-\mathbf{k}, i \omega_{v}-i \omega_{m}\right) \\
& =\frac{1}{V} \sum_{\mathbf{k}} \frac{1-f\left(\varepsilon_{\mathbf{q} / 2+\mathbf{k}}\right)-f\left(\varepsilon_{\mathbf{q} / 2-\mathbf{k}}\right)}{\omega_{v}+2 \mu-\varepsilon_{\mathbf{q} / 2+\mathbf{k}}-\varepsilon_{\mathbf{q} / 2-\mathbf{k}}}
\end{aligned}
$$

In Eq. (52) the Matsubara frequencies $\omega_{m}=m 2 \pi T i$ have been summed over integers $m$.

We now scale the potential by $\lambda$ and sum up interactions energies $(U \Pi)^{n}$ from $n=1,2, \ldots$ ladders. We obtain for the expectation value of the interaction energy in state $\lambda$ gives

$$
\langle\lambda U\rangle=-T \sum_{\mathbf{q}, \omega_{v}} \frac{\lambda U \Pi\left(\mathbf{q}, \omega_{v}\right)}{1-\lambda U \Pi\left(\mathbf{q}, \omega_{v}\right)} .
$$

From the Hellmann-Feynmann theorem we now obtain the interaction part of the free energy

$$
\Omega_{\text {int }}=\int_{0}^{1} \frac{d x}{\lambda}\langle\lambda U\rangle=T \sum_{\mathbf{q}, \omega_{v}} \ln \left[1-U \Pi\left(\mathbf{q}, \omega_{v}\right)\right]
$$

As in the gap equation (42) large momenta contributions are removed by replacing $U \Pi$ by $U_{0} \Pi_{r}$, where the renormalized propagator is

$$
\Pi_{r}\left(\mathbf{q}, \omega_{v}\right)=\Pi\left(\mathbf{q}, \omega_{v}\right)+\frac{1}{V} \sum_{\mathbf{k}} \frac{1}{2 \varepsilon_{\mathbf{k}}} .
$$

Inserting into Eq. (54) gives the thermodynamic potential

$$
\Omega=\Omega_{0}+T \sum_{\mathbf{q}, \omega_{v}} \ln \left[1-U_{0} \Pi_{r}\left(\mathbf{q}, \omega_{v}\right)\right],
$$

where $\Omega_{0}=-2 T \sum_{\mathbf{q}} \ln \left[1+e^{\left(\varepsilon_{q}-\mu\right) / T}\right]$ is the free energy for non-interacting Fermions. The frequency sum in Eq. (56) can, using the residue theorem, be converted to an 
$\omega$-integral of the Bose distribution function $(\exp (\omega / T)-1)^{-1}$ around the real axis, where the logarithm in Eq. (56) has a cut.

In the BEC limit, where $\mu$ is large and negative, it follows from Eq. (52) that $1-U_{0} \Pi_{r}(\mathbf{q}, \omega)$ is proportional to $\omega-\varepsilon_{M}(q)+2 \mu$, where

$$
\varepsilon_{M}(q)=-\frac{\hbar^{2}}{m a^{2}}+\frac{\hbar^{2} q^{2}}{4 m}
$$

is the molecular binding and kinetic energy of a pair with mass $2 m$ and momentum q. Therefore the derivative of $\ln \left[1-U_{0} \Pi_{r}\left(\mathbf{q}, \omega_{v}\right)\right]$ with respect to $\mu$ has a pole at $\omega=\varepsilon_{M}(q)-2 \mu$ with residue 2 . Using the residue theorem again we obtain the number equation

$$
N \simeq-\frac{d \Omega}{d \mu} \simeq 2 \sum_{\mathbf{q}} \frac{1}{e^{\left(\varepsilon_{M}(q)-2 \mu\right) / T}-1}
$$

Here we have ignored the contribution to the thermodynamic potential $\Omega_{0}$ from free Fermions which is negligible in the BEC limit. The number equation is simply that of a free Bose gas as opposed to that of a Fermi gas Eq. (48) in the BCS limit.

Whereas the number equation changes qualitatively from free Fermi atoms to free Bose molecules in the crossover, the gap equation is unchanged and given by Eq. (42). In the BEC limit the gap equation simply yields that the chemical potential is half the molecular binding energy, $\mu=-\hbar^{2} / 2 m a^{2}$. The number equation now gives

$$
T_{c}=\frac{\pi}{[2 \zeta(3 / 2)]^{2 / 3}} \frac{\hbar^{2} n^{2 / 3}}{m} \simeq 0.218 E_{F},
$$

i.e., the critical temperature is independent of the pairing interaction and given by the $T_{c}^{B E C}$ for the molecular BEC. At $T_{c}^{B E C}$ all bosons are thermally excited with none remaining at zero momentum.

In general the gap equation (58) and number conservation $N=d \Omega / d \mu$ with $\Omega=\Omega_{0}+\Omega_{\text {int }}$ from Eq. (54) have to be solved self-consistently for the critical temperature and the chemical potential in the BCS-BEC crossover. The result for $T_{c}$ is shown in Fig. (5). One notices that NSR predicts a maximum near the unitarity limit. Recent experiments find $T_{c} / E_{F}=0.19 \pm 0.2$ [20] whereas earlier found $T_{c} / E_{F}=0.29 \pm 0.3$ [4]. This is compatible with the gap $\Delta=0.44 E_{F}$ found in [55].

Although the NSR model is a qualitative improvement of the Leggett model by correctly describing $T_{c}$ in the BEC limit, it still omits a number of effects such as particle-hole contributions, selfenergies and induced interactions from the medium. Consequently, the Hartree field and Gorkov corrections are not included, and in the BEC limit the molecule-molecule scattering length $2 a$ implied by Eq. (50) is a factor $\sim 3$ too large [54]. More elaborate models [56, 57, 58] include some of these effects and do not find a maximum for $T_{c} / E_{F}$ around the unitarity limit. 


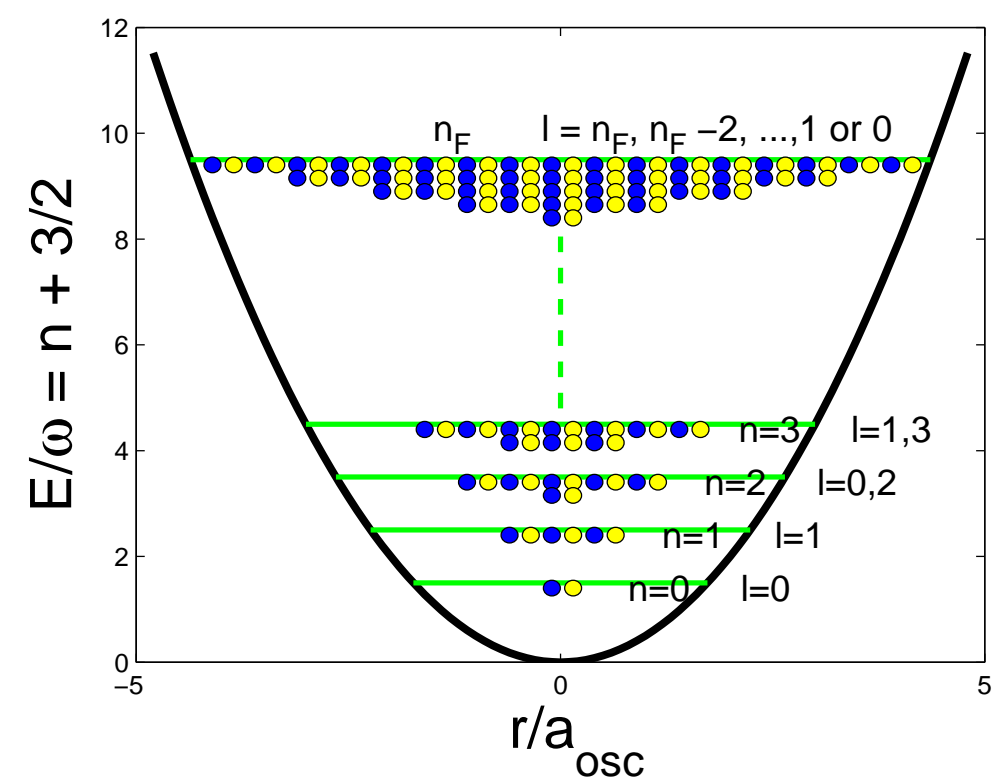

Fig. 6 Shells in a 3D harmonic oscillator trap.

\section{Atomic traps and Nuclei}

Traps are necessary for confining the atoms and creates a density distribution that is maximal at central density and decrease towards the cloud size $R$ where it vanishes. Thus all densities are present at once which can be difficult to separate experimentally in order to extract the $(\mathrm{n}, \mathrm{T})$ phase diagram.

From a nuclear point of view harmonic oscillator traps are wonderful toy systems since they provide confined systems, which are a first approximation to the nuclear mean field potential. Additionally we can tune the interactions and therefore the level splitting and pairing. Also millions of Fermi atoms can be confined and not just the $\lesssim 250$ neutron and protons in nuclei limited by fission. Therefore one can study the crossover from few to infinite number of particles which is necessary in order to e.g. link pairing in nuclei to that in neutron and nuclear matter. Similarities between the (prolate) nuclear mean field and cigar shaped optical traps can also be exploited in studies of shell structures, pairing and collective modes. 


\subsection{Scaling in atomic traps}

In a noninteracting two spin balanced Fermi gas in a spherically symmetric HO potential at zero temperature the particle fill $\mathrm{HO}$ shells of energy $n \hbar \omega, n=1,2, . ., n_{F}$ up to the Fermi shell $n_{F}=(3 N)^{1 / 3}$ with Fermi energy $E_{F}=n_{F} \hbar \omega$. For a sufficiently large number $N$ of particles confined in a (shallow) trap the system size $R$ is so long that density variations and the extent of possible phase transition interfaces can be ignored and one can apply the local density and the Thomas-Fermi approximation. Here the total chemical potential is given by the sum of the harmonic oscillator (HO) trap potential and the local chemical potential $\mu_{i}=d E / d N_{i}$

$$
\mu_{i}(r)+\frac{1}{2} m \omega^{2} r^{2}=\frac{1}{2} m \omega^{2} R_{i}^{2}
$$

which must be constant over the lattice for all components $i=1,2, \ldots$ It can therefore be set to its value at the its edge $R_{i}$, which gives the r.h.s. in Eq. 60). We shall mainly discuss spin balanced two component systems where the chemical potential and radii are equal. The equation of state determines $\mu(n)$ in terms of the universal function of Eq. 60).

In the dilute limit and in the unitarity limits $\mu=\xi \hbar^{2} k_{F}^{2} / 2 m$, where $\xi=1$ and $\xi=1+\beta(0)$ respectively. In both cases Eq. (60) gives $n(r)=k_{F}^{3}(r) / 3 \pi^{2}=$ $n_{0}\left(1-r^{2} / R^{2}\right)^{3 / 2}$, where $n_{0}=\xi^{-3 / 4}\left(2 n_{F}\right)^{3 / 2} / 3 \pi^{2} a_{o s c}^{3}$ is the central density and $R=\xi^{1 / 4} \sqrt{2 n_{F}} a_{o s c}$ the cloud size; $a_{o s c}=\sqrt{\hbar / m \omega}$ is the oscillator length. The attraction contracts the gas to a higher central density such that $R \propto k_{F}^{-1}(0) \propto \xi^{1 / 4}$. The total energy of the trapped gas is $E / N=(3 / 8) \xi^{1 / 2} n_{F} \hbar \omega$.

For a more general equation of state $P \propto n^{\gamma+1}$ or $\mu \propto n^{\gamma}$ with polytropic index $\gamma$ the equilibrium density is $n_{e q}=n_{0}\left(1-r^{2} / R^{2}\right)^{1 / \gamma}$, where $R^{2}=2(\gamma+1) P_{0} / \gamma n_{0} m \omega^{2}$. $P_{0}$ and $n_{0}$ are the pressure and density in the centre of the trap. In both the noninteracting and the unitary limit $\gamma=2 / 3$ whereas $\gamma=1$ in the BEC limit and for a bose gas.

As suggested in [12] it is convenient to measure the density along the axial axis integrated over transverse cross section $n(z)=\int n(r) d x d y$. From the Gibbs-Duhem equation $(d P / d \mu)_{T}=n$ using Eq. 60) we obtain the pressure in the centre along the axial axis by integrating over transverse coordinates

$$
P(z)=\frac{m \omega_{\perp}}{2 \pi} n(z)
$$

At the same time the chemical potential $\mu(z)=m \omega_{z}\left(R_{z}^{2}-z^{2}\right)$ is known by measuring the size $R(z)$ of the cloud along the $z$-axis. Thus $P(\mu)$ can be measured and the equation of state extracted at any temperature.

In a Fermi liquid we obtain from Eqs. (9) and (14) that the pressure w.r.t. that in a free Fermi gas is

$$
P(x, T) / P_{F G}=1+\beta(x)+\frac{5 \pi^{2}}{8} \frac{m^{*}}{m} \frac{T^{2}}{E_{F}^{2}} .
$$


Detailed measurements of the intensive variables $(T, P, \mu)$ gives $\beta(0)=-0.49(2)$ and $m^{*} / m=1.13(3)$ [20].

\subsection{Collective modes}

Tickling the trapped atoms sets them into oscillations at certain eigen-frequencies called collective modes. Such giant dipole and quadrupole modes have been important for studying nuclei [59]. The collective modes can be calculated from the equation of state and the Euler equation

$$
m n \frac{\partial \mathbf{v}}{\partial t}=-\nabla\left(P+n m \sum_{i} \omega_{i}^{2} r_{i}^{2}\right) .
$$

where $\mathbf{v}=\partial \mathbf{r} / \partial t$ is the local velocity. The last term is the gradient of a generally deformed $\mathrm{HO}$ potential. The Euler equation can be solved analytically for polytropic equation of states. For spherical symmetric 3D traps one finds collective modes at eigen frequencies $\omega_{\eta l}$ for a mode with $\eta=0,1,2, \ldots$ nodes and angular momentum $l$ given by [60]

$$
\frac{\omega_{\eta l}^{2}}{\omega_{0}^{2}}=l+2 \eta[\gamma(\eta+l+1 / 2)+1]
$$

Similar expressions exist for modes in deformed traps [61] which have been measured in detail [4, 17].

For attractive interactions the system is superfluid at zero temperature and therefore irrotational with a quenched moment of inertia. As the temperature is increased above $T_{c}$ the moment of inertia increase to rigid value and hereby the critical temperature $T_{c}=0.19(1) E_{F}$ is found [62]. Deformed system can be rotated but has a quenched moment of inertia $I=\delta^{2} I_{\text {rigid }}$ [63].

\subsection{Shell structure and pairing in atomic traps}

The pairing gap generally increase with the level degeneracy or density of states [59]. For pairing in a single level of angular momentum $l$ or shell $n_{F}$ the pairing gap scales with the number of degenerate states $(2 l+1)$ or $\left(n_{F}+1\right)\left(n_{F}+2\right)$ respectively. If levels are split or distributed the gap depends on the density of levels around the Fermi level as in the uniform case of Eq. 443).

For very weak interactions the level splitting of single particle levels $l=n_{F}, n_{F}-$ $2, \ldots, 1$ or 0 in the HO shell $n_{F}$ is smaller than the pairing gap when there are sufficiently few $\left(n_{F} \lesssim 10\right)$ atoms in a $\mathrm{HO}$ potential. The pairing occurs between all states in the shell leading to the supergap [65] 


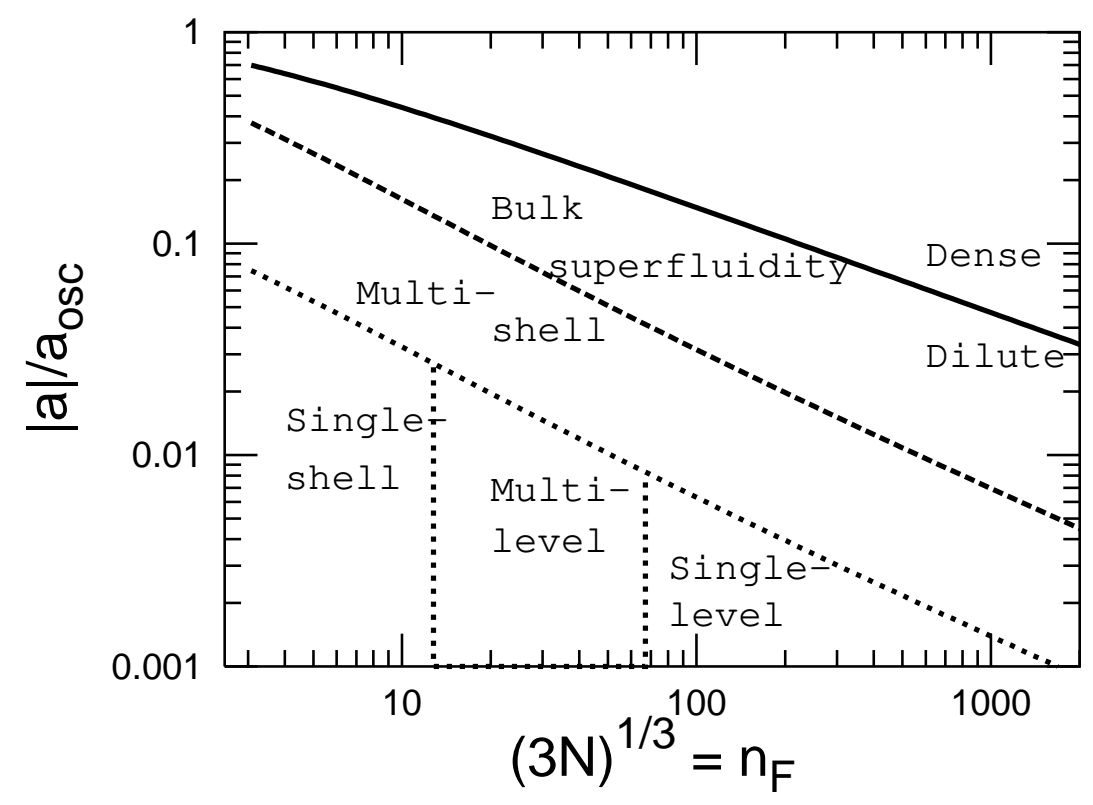

Fig. 7 Diagram displaying the regimes for the various pairing mechanisms (see text) at zero temperature in h.o. traps vs. the number of particles $N=n_{F}^{3} / 3$ and the interaction strength $a$. The dotted lines indicate the transitions between single-shell pairing $\Delta=G$, multi-level, single-level, and multi-shell pairing. At the dashed line determined by $2 G \ln \left(\gamma n_{F}\right)=\hbar \omega$ the pairing gap is $\Delta \simeq \hbar \omega$, and it marks the transition from multi-shell pairing to bulk superfluidity Eq. (45). The pairing gap is $\Delta=0.54 E_{F}$ above the full line $\rho|a|^{3} \geq 1$, which separates the dilute from the dense gas. From [65]

$$
G=\frac{32 \sqrt{2 n_{F}+3}}{15 \pi^{2}} \frac{|a|}{a_{o s c}} \hbar \omega .
$$

For more particles or stronger interactions the situation becomes more complicated depending on the gap size with respect to the level splitting as shown in Fig. (7). Increasing the number of particles cause level splitting so that pairing is reduced to multi-level and eventually single level pairing. Increasing the interaction strength increase pairing to nearby shells [65] referred to as multi-shell pairing in Fig. (7). The general case can be solved within the Bogoliubov-deGennes equations [65, 31]. For stronger interactions and many particles the pairing approaches that in a uniform or bulk system, and eventually dense system with $|a| k_{F}>1$ and the unitarity limit is reached.

The various pairing mechanisms and phases of Fig. (7) can be studied at low temperatures is which hopefully will be reached in the near future. 


\subsection{Pairing in nuclei}

We can exploit some interesting similarities between pairing in nuclei [59, 64] and that of Fermi atoms in traps [65, 31]. To a first approximation the nuclear mean field is often taken as harmonic oscillator (HO) potential just as the optical traps. Secondly, the residual pairing interaction between nucleons is taken as a short range (delta function) interaction as for cold atoms.

Before these result can be applied to nuclei there are, however, a number of differences that must be taken into account. Large nuclei have approximately constant central density $\rho_{0} \simeq 0.14 \mathrm{fm}^{-3}$ and Fermi energy $E_{F}$ in bulk. Therefore the HO frequency, which is fitted to the nuclear mean field, decreases with the number of nucleons $A=N+Z$, where $N$ now is the number of neutrons and $Z$ the number of protons in the nucleus, as $\hbar \omega \simeq E_{F} / n_{F} \simeq 41 \mathrm{MeV} / A^{1 / 3}$. In the valley of $\beta$-stability the number of protons is $Z \simeq A /\left(2+0.0155 A^{2 / 3}\right)$. Therefore in medium and large nuclei the difference between the Fermi energies of protons and neutrons exceeds the pairing gap so that pairing between protons and neutrons does not occur.

Secondly, the nuclear mean field deviates from a $\mathrm{HO}$ potential by being almost constant inside the nucleus and vanish outside. The resulting net anharmonic nuclear field is stronger and opposite in sign to the corresponding (anharmonic) mean field in atomic traps. Therefore, the level splitting is larger and the ordering of the 1levels is reversed. In addition, a strong spin-orbit force splits the single particle states of total angular momentum $j=l \pm 1 / 2$, such that the $j=n_{F}+1 / 2$ is lowered down to the shell $\left(n_{F}-1\right)$ below. The level splitting can be parametrized by a single parameter taken from analyses of nuclear spectra. It increases with shell number up to $n_{F} \simeq 6$ for heavy nuclei. Due to the strong spin-orbit force the $j=l \pm 1 / 2$ states are split and the $j=n_{F}+1 / 2$ is lowered down to the shell below. The magic numbers become $N, Z=8,14,28,50,82,126,184, .$. , etc. rather than the h.o. filled shell particle numbers $N, Z=2,8,20,40,70,112,168,240, \ldots$, etc.

The pairing gaps and quasi-particle energies can now be calculated by solving the Bogoliubov-deGennes gap equation [65] which are shown in Fig. (8) for neutrons. The strong level splitting in nuclei has the effect that pairing is strongest when the shell is half filled and weak near closed shells simply because there are fewer states available for pairing, i.e., the level density is smaller. Averaging over several shells, however, the mean gap is well approximated by the supergap because the reduction in pairing due to level splitting is compensated by additional pairing to nearby shells. Since $\hbar \omega$ scale as $\sim A^{-1 / 3}$ and $a_{o s c} \propto n_{F}^{1 / 2}$ the single-shell pairing gap also scales as $G \sim A^{-1 / 3}$. Therefore, the pairing gaps in light and medium mass nuclei scale approximately as [31]

$$
\Delta \simeq G \simeq \frac{|a|}{0.41 \mathrm{fm}} \frac{5.5 \mathrm{MeV}}{A^{1 / 3}}
$$

As shown in Fig. (8) the supergap does not depend on the level-splitting and is therefore a robust prediction for the average magnitude and mass scaling of pairing gaps in nuclei. 


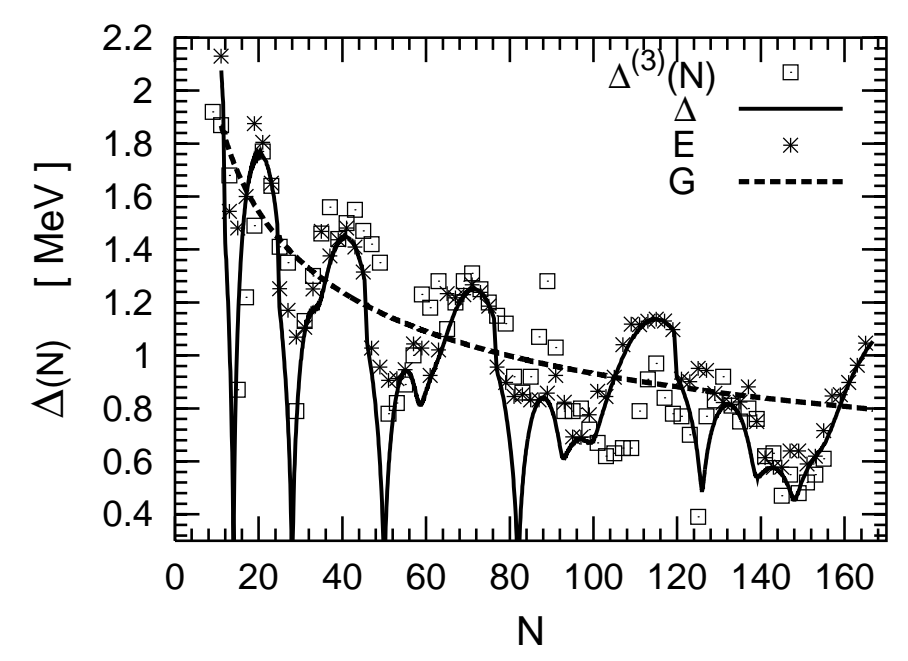

Fig. 8 Neutron pairing energies vs. the number of neutrons. The experimental odd-even staggering energies $\Delta^{(3)}(N)$ are averaged over isotopes [67 66]. The calculated gaps $\Delta$ and quasi-particle energies $E$ are obtained from the gap equation (see text) with effective coupling strength $a=$ $-0.41 \mathrm{fm}$. The supergap $G$ is shown with dashed line. See [31] for details and corresponding plot for protons.

The data on neutron and proton pairing is obtained from the odd-even staggering of nuclear binding energies $B(N, Z)$. It has been shown that mean field contributions can be removed [66] by using the three-point filter $\Delta^{(3)}(N)=(-1)^{N}[B(N-$ $1, Z) / 2+B(N+1, Z) / 2-B(N, Z)]$. We compare in Fig. (8) to the experimental $\Delta^{(3)}(N)$ averaged over isotopes with the calculated gaps. The analogous for protons $\Delta^{(3)}(Z)$ averaged over isotones compare similarly to Bogolibov-deGennes calculations [65, 31]. In the calculations the effective coupling is the only adjustable parameter which is fitted to the experimental data. For both neutrons and protons we extract $a \simeq-0.41 \mathrm{fm}$.

Considering the simplicity of the model it describes a large number of experimental gaps fairly well on average. In a number of cases, however, the calculated pairing gaps differ significantly from the measured neutron gaps. Some of these deviations can be attributed to the crude single particle level spectra assumed.

The pairing in nuclear matter can now be estimated once the effective interaction has been determined using Eq. (43). Note that the effective interaction includes induced interactions. Inserting $a=-0.41 \mathrm{fm}$ and $k_{F}=1.3 \mathrm{fm}^{-1}$ at nuclear saturation density, $\rho_{0}=0.15 \mathrm{fm}^{-3}$, we obtain the proton and neutron pairing gaps

$$
\Delta \simeq 1.1 \mathrm{MeV},
$$

in the bulk of very large nuclei and in symmetric nuclear matter at nuclear saturation density. This number is compatible with earlier calculations [39] of the ${ }^{1} S_{0}$ pairing gap in nuclear and neutron star matter around normal nuclear matter densities. 
Neutron star matter has a wide range of densities and is asymmetric, $Z / A \sim 0.1$, above normal nuclear matter densities. One can attempt to estimate of the pairing gaps as function of density from the gap in bulk, Eq. [43), with $a \simeq-0.41 \mathrm{fm}$ and the neutron or proton Fermi wave numbers $k_{F}^{N, Z}=\left(3 \pi^{2} \rho_{N, Z}\right)^{1 / 3}$ as function of densities. However, the effective interaction $a$ is density dependent. At higher densities we expect that the effective interaction becomes repulsive as is the case for the nuclear mean field at a few times nuclear saturation density. At lower densities the effective scattering length should approach that in vacuum which for neutronneutron scattering is $a\left({ }^{1} S_{0}\right) \simeq-18 \mathrm{fm}$.

In spite of several simplifying approximations in this atomic trap model for nuclear pairing it provides at least a qualitative description of pairing in most nuclei. The effective interaction $a=-0.41 \mathrm{fm}$ is the same for both neutrons and protons which reflects that both are close to forming bound states. At central densities $x=1 / a k_{F} \simeq-1.9$, i.e. on the BCS side of the unitarity limit.

\subsection{Quark and gluon matter}

The Coulomb and QCD interactions $\sim g^{2} / r$ or their Fourier transform $\sim g^{2} / q^{2}$ are long range and therefore contrary to the short range interactions underlying universality. The long range QED and QCD interactions requires screening of infrared divergences [68] whereas in unitary gases ultraviolet cutoffs are provided by renormalizing the short range interaction in terms of the scattering length. Never the less a number of similarities have appeared.

Confinement is caused by strong color fields between quarks and gluons which forms bound state hadrons at temperatures below $T \lesssim T_{Q G P} \simeq 160 \mathrm{MeV}$ and densities below a few times normal nuclear matter density, $\rho_{0}=0.15 \mathrm{fm}^{-1}$. These may again form a nuclear liquid at temperatures below the nucleon gas critical temperature $T \lesssim T_{N G} \simeq 15 \mathrm{MeV}$ for a four component nuclear system as discussed above. In the hadron gas the interparticle distance is larger than the confinement interaction range, and the quark molecules can therefore be viewed as a molecular gas. However, color neutrality requires either Fermi molecules of three quarks (baryons) or quark-antiquark Bose pairs (mesons). Of the two phase transitions in the molecular gas of hadrons only the neutron gas can be viewed as a BCS-BEC crossover as discussed in sec. (2.6). The instability and first order transition of a four-component nuclear gas was discussed in sec. (2.6) can be viewed as multi-component crossover. Here the effective scattering length is that between neutrons and protons $a \sim 20 \mathrm{fm}$, i.e. on the molecular BEC side, and the nuclear matter is in the unitarity limit in the sense that $x=1 / a k_{F} \sim 0_{+}$although they now are kept in place by short range repulsive forces. The transition from nuclear to quark matter or a hadron gas to a quark-gluon plasma is quite different because the interaction range is always of order the interparticle distance. The strong short range repulsive forces between nucleons leads to a strongly correlated wavefunction as in hard sphere scattering and the strongly correlated nuclear fluid. In this case the Gutzwiller approximation of 
Ref. [46] is valid and the matter does not undergo a ferromagnetic transition separating the unlike components (see Fig. (1)). As seen from the quark-gluon side one can define a "crossover parameter" $x \sim \Lambda_{Q C D} / k_{F}$ which at high densities is in the unitarity limit. With decreasing densities the running coupling constant $\Lambda_{Q C D}$ diverges when the quark-gluon plasma undergoes a first order transition to a hadron gas with $x \gtrsim 1$.

Quark pairing also have similarities to pairing in nuclear and multi-component atomic gases. Color superconductivity between the 2 spin, 8 color and 2-3 flavor states is very sensitive to flavor imbalance [8]. If the strange quark mass is small the up, down and strange quark Fermi levels are close. If pairing is sufficiently strong such that the gap exceeds the Fermi level splitting all flavors can pair. It is amazing that properties of pairing in quark matter, which may exist in unaccessible cores of neutron stars, can be studied in tabletop experiments with ultracold multicomponent spin-imbalanced Fermi atomic gases.

Another interesting similarity is elliptic flow. The overlap zone in semi-central high energy nuclear collisions is prolate (cigar shaped) as are optical traps. In subsequent expansion the hydrodynamics forces stronger expansion in the direction where systems is narrowest initially. This makes the momentum distribution azimuthally asymmetric - referred to as elliptic flow. Results indicate that both "liquids", the QGP/hadronic and ultracold unitary atomic gas, expand as almost perfect fluids initially i.e. their viscosities are record breaking low [69].

Correlations can reveal the quantum phase structure. Originally Hanbury-Brown \& Twiss measured the Bose-Einstein correlations between stellar photons and determined the diameters of nearby stars. Similar correlations between mesons in high energy nuclear collisions have been exploited to determine the freezeout size of the collision zones [70]. Also Fermi anticorrelations have been observed between baryons. In ultracold atomic systems analogous "noise" correlations have been found near the BCS-BEC crossover due to pairing [16]. Bragg peaks have been observed for bosons in 3D [71] and 2D [72] lattices, and dips for 3D fermions in [73].

\section{Optical Lattices}

A standing wave can be generated by shining lasers onto traps which generates a $3 \mathrm{D}$ cubic or $2 \mathrm{D}$ square lattice potential with spacing $\lambda=2 \pi / k$, which is half the laser wavelength. The lattice height $V_{0}$ varies with laser intensity and the scattering length. In the tight binding approximation the onsite coupling

$$
U=E_{R} a k \sqrt{8 / \pi} \xi^{3}
$$

and hopping parameter

$$
t=E_{R}(2 / \sqrt{\pi}) \xi^{3} e^{-2 \xi^{2}}
$$



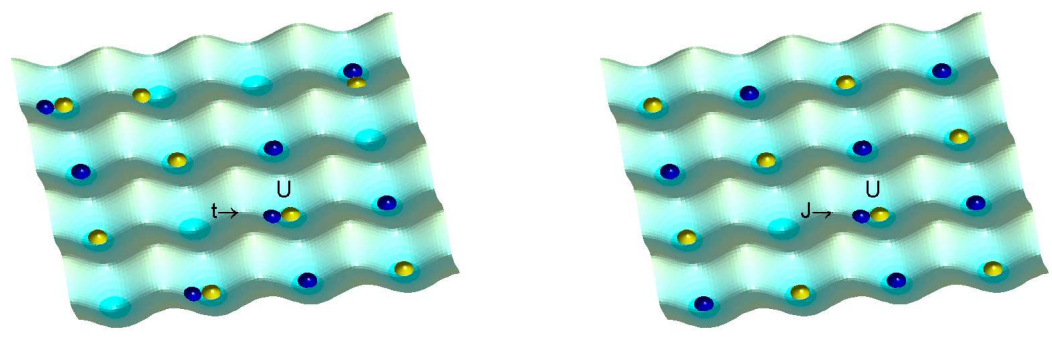

Fig. 9 2D optical lattices loaded with fermions in two spin states (yellow and blue) at half filling. For onsite attraction $(U<0$, left) opposite spins tend to pair. For onsite repulsion $(U>0$, right) the ground state is an $\mathrm{AF}$ with virtual hopping $J=4 t^{2} / U$.

where $E_{R}=\hbar^{2} k^{2} / 2 m$ is the recoil energy and $\xi=\left(V_{0} / E_{R}\right)^{1 / 4}$. Varying $V_{0}$ and the scattering length $a$ near Feshbach resonances allows us to tune the Hubbard parameters $U$ and $t$.

The lattice constant $\lambda$ introduces another lengths scale so that the thermodynamic quantities generally depend on both density and interaction strength. Therefore universality is broken except for low filling which corresponds to dilute Fermi gases.

The Hubbard Hamiltonian on a D-dimensional lattice describes optical lattices that are sufficiently deep for a one-band model to apply,

$$
H=\sum_{i, \sigma<\sigma^{\prime}} U_{\sigma, \sigma^{\prime}} \hat{n}_{i \sigma} \hat{n}_{i \sigma^{\prime}}-t \sum_{\langle i j\rangle, \sigma} \hat{a}_{i \sigma}^{\dagger} \hat{a}_{j \sigma} .
$$

Here $\hat{a}_{i \sigma}^{\dagger}$ is the Fermi creation operator of the hyperspin states $\sigma=1,2, \ldots, v, n_{i \sigma}=$ $\hat{a}_{i \sigma}^{\dagger} \hat{a}_{i \sigma}$ the density and $\langle i j\rangle$ denotes nearest neighbours with hopping parameter $t$. Due to particle-hole symmetry results for a given site filling $n$ also applies to $2-n$.

The Hubbard model is of fundamental importance in condensed matter physics where it explains properties of Mott insulators, antiferromagnets, d-wave superconductivity, etc. The Hubbard model can only be solved in 1D for fermions but a number of models and numerical calculations have given insight in the various phases and order parameters. Optical lattices may tell us the answers in a few years.

\subsection{On-site attraction and Pairing}

We start by investigating s-wave pairing between to spin balanced states due to an attractive on-site interaction $U<0$. The mean field gap equation for singlet superfluidity at zero temperature is [74] 


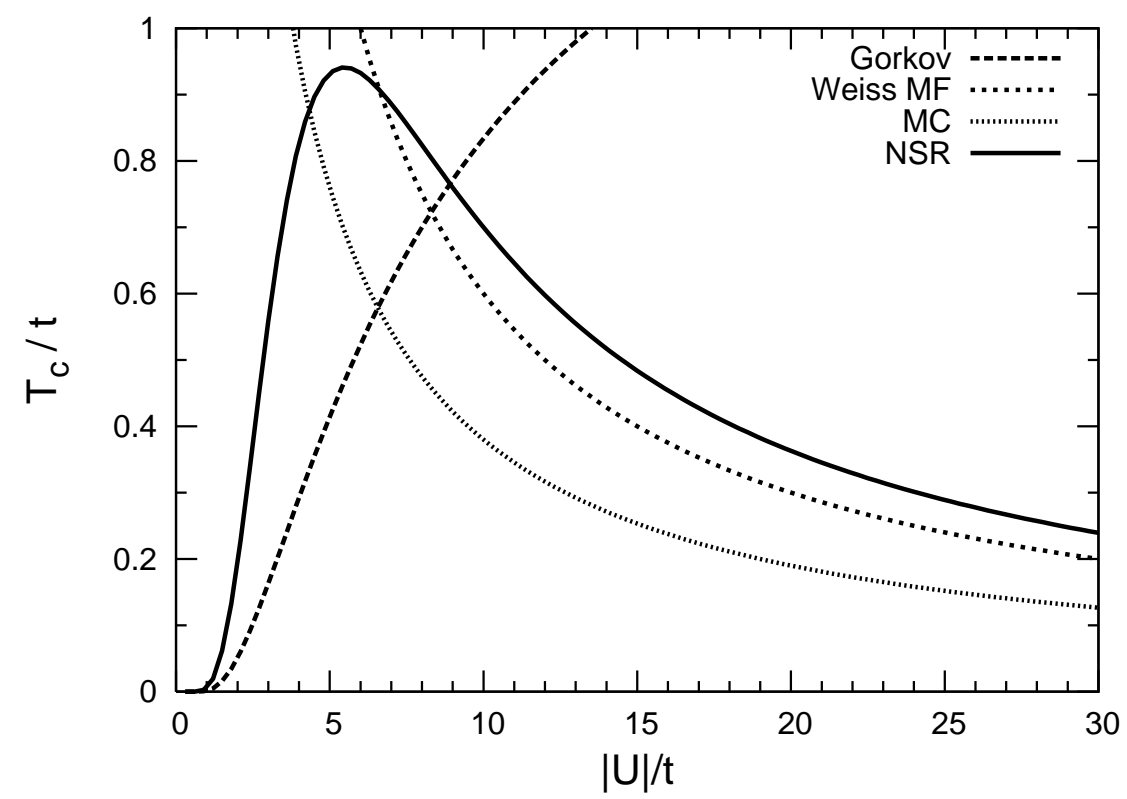

Fig. 10 Superfluid transition temperatures in a 3D lattice at half filling for the NSR model, the Heisenberg model within (Weiss) mean field $T_{c}=1.5 \mathrm{~J}$ and Monte Carlo. "Gorkov" is the weakly attractive limit of Eq. (74) reduced by a factor 3.9 due to induced interactions.

$$
\Delta_{\mathbf{p}^{\prime}}=-\frac{1}{M} \sum_{\mathbf{p}} U\left(\mathbf{p}^{\prime}, \mathbf{p}\right) \Delta_{\mathbf{p}} \frac{\tanh \left(E_{\mathbf{p}} / 2 T\right)}{2 E_{\mathbf{p}}}
$$

where $M$ is the number of lattice points and $E_{\mathbf{p}}=\sqrt{\left(\varepsilon_{\mathbf{p}}-\mu\right)^{2}+\Delta_{\mathbf{p}}^{2}}$ with $\varepsilon_{\mathbf{p}}=$ $2 t \sum_{i=1, D}\left(1-\cos p_{i}\right)$.

The density $n=1-\sum_{\mathbf{p}}\left(\varepsilon_{\mathbf{p}} / E_{\mathbf{p}}\right) \tanh \left(E_{\mathbf{p}} / 2 T\right) / M$, is also the filling fraction in units where the lattice constant is unity. Momenta are in the first Brillouin zone only $\left|p_{i}\right| \leq \pi$ taking $\lambda=1$ for convenience.

Due to particle-hole symmetry results also apply replacing the density by $(2-n)$ and chemical potentials by $(4 D t-\mu)$, where $4 D t$ is the bandwidth in $D$ dimensions. At low filling $\varepsilon_{p}=t p^{2}$ and results also apply to uniform systems replacing $t=1 / 2 m$.

\subsubsection{Pairing in the dilute limit}

We first calculate the zero temperature gaps for weak attraction $|U| \ll t$ excluding induced interactions. The s-wave gap $\Delta_{\mathbf{p}^{\prime}}=\Delta_{0}$ is then momentum-independent because the interaction $U$ is. The gap equation (71) reduces to: $1=-\left(U_{0} / M\right) \sum_{\mathbf{p}} 1 / 2 E_{\mathbf{p}}$, from which the gap can be calculated analytically at low and half filling to leading orders in 3D and 2D (for 1D see, e.g. [75]). 
The 3D lattice has a critical coupling [76]

$$
U_{c}=-M /\left(\sum_{\mathbf{q}} 1 / 2 \varepsilon_{\mathbf{q}}\right)=-8 \sqrt{2} t /\left[\sum_{l=0}^{\infty} P_{2 l}(\sqrt{9 / 8})(2 l-1) ! ! / 2^{2 l} l !\right] \simeq-7.913 t,(72)
$$

where a two-body bound state can be formed at zero density $(\mu=0)$. This threshold corresponds to the unitarity limit of infinite scattering length in the uniform system and naturally enters the sum in the gap equation. The dilute gap becomes to leading orders

$$
\Delta_{0}^{3 D}=\frac{8}{e^{2}} \mu \exp \left[\frac{4 \pi^{2} t}{k_{F}}\left(\frac{1}{U}-\frac{1}{U_{c}}\right)\right], n \ll 1,
$$

at low densities where $\mu=t k_{F}^{2} \ll t$. The level density at the Fermi surface $N(\mu)=$ $k_{F} /\left(4 \pi^{2} t\right)$ enters in the exponent as in standard BCS theory. $U_{c}$ acts as a cutoff in the one-band Hubbard model which in the uniform (continuum) limit is absorbed into the scattering length $a$. Thus Eq. (73) is the finite lattice equivalent of the gap in the uniform system $\Delta_{0}^{3 D}=\left(8 / e^{2}\right) \mu \exp \left(\pi / 2 k_{F} a\right)$, with $U^{-1}-U_{c}^{-1}=U_{0}^{-1}$, where $U_{0}=4 \pi a / m$ as in the uniform case, such that threshold $U=U_{c}$ corresponds to $|a|=\infty$.

Near half filling the level density is surprisingly constant $N(\mu) \simeq 0.143 / t$ in a wide range $4 t \leq \mu \leq 8 t$ around half filling. To leading orders the gap becomes [74]

$$
\Delta_{0}^{3 D}=\alpha t \exp \left[\frac{1}{N(\mu) U}\right], n \simeq 1,
$$

where the prefactor $\alpha \simeq 6.544$ can be calculated numerically.

The 2D lattice has a superfluid s-wave gap that be calculated from the gap equation [77]

$$
\Delta_{0}^{2 D}=\sqrt{8 \pi \mu t} \exp \left[\frac{4 \pi t}{U}\right], n \ll 1,
$$

when the density $n=k_{F}^{2} / 2 \pi$ is small. The $2 \mathrm{D}$ level density is $N(\mu)=1 /(4 \pi t)$. Eq. (75) assumes that the on-site coupling and the gap are small such that $\Delta_{0}^{2 D} \lesssim \mu$. This is not fulfilled at sufficiently low densities where instead $\Delta_{0}^{2 D}=4 \pi t \exp (8 \pi t / U)$, which also is the two-body binding energy in $2 \mathrm{D}$ at zero density. There is always a two-body bound state in $2 \mathrm{D}$ with purely attractive interaction and therefore $U_{c}$ vanishes. In 3D a similar pair condensate (a molecular BEC) appears when $a>0$ corresponding to $U<U_{c}$.

For intermediate fillings the pairing is more complicated in 2D. Near half filling $n \simeq 1$ the level density $N(\varepsilon)=\ln (16 t /|\varepsilon-4 t|) /\left(2 \pi^{2} t\right)$ has a logarithmic singularity due to the van Hove singularity. Calculating the r.h.s. of the gap equation therefore gives a double log: $1=|U| /\left(4 \pi^{2}\right) \ln ^{2}\left(32 t / \Delta_{0}^{2 D}\right)$, to leading logarithmic orders, resulting in the gap when $n \simeq 1$ [77] 


$$
\Delta_{0}^{2 D}=32 t \exp (-2 \pi \sqrt{t /|U|}) .
$$

In the strong coupling limit $\Delta=|U| / 2$.

\subsubsection{Induced interactions on the lattice}

In $3 \mathrm{D}$ the induced interactions are at low densities $n \ll 1$ the same as in the uniform case Eq. (44) and reduce gaps and $T_{c}$ by a factor $(4 e)^{1 / 3} \simeq 2.2$. Near half filling the reduction factor is $\sim 3.9$ for weak interactions. For stronger interactions the induced interactions are enhanced [52] leading to a reduction of the gap by a factor $\sim 25$ when $U=-3 t$.

In $2 \mathrm{D}$ the induced interactions suppress the gap by a factor $e$ in the dilute limit [78] but are divergent at half filling due to the singular level density [52]. The induced interactions have been calculated for general spin polarization and number of components in 3D and 2D optical lattices [53].

\subsubsection{Crossover and the BEC limit}

As the interactions become stronger the Fermi gas gradually undergo crossover to a molecular BEC. On the lattice, however, the finite bandwidth automatically provides a cutoff which affects the crossover and changes the BEC limit on the lattice qualitatively from the uniform case. We shall describe this crossover employing the NSR model as above for the uniform case but with a finite bandwidth. Our starting point is again the two-particle correlation function of Eq. 52. In the BEC limit (large negative chemical potential) we find from $\mathrm{Eq}$. (52) that $1-U \Pi(\mathbf{q}, \omega)$ is proportional to $\omega-\varepsilon_{M}(q)+2 \mu$ as in NSR, where now the molecular boson energy is

$$
\varepsilon_{M}(\mathbf{q})=-U+J \sum_{i=1,3}\left[1-\cos \left(q_{i}\right)\right]
$$

with $J=4 t^{2} / U$. Therefore the number equation reduces to the condition for BEC in an ideal Bose gas as Eq. (58)

$$
N \simeq-\frac{d \Omega_{i n t}}{d \mu} \simeq 2 \sum_{\mathbf{q}} \frac{1}{e^{\left(\varepsilon_{M}(\mathbf{q})-2 \mu\right) / T}-1},
$$

but with the molecular boson energy of Eq. (77) in stead of 57. The gap equation at $T_{c}$ yields a chemical potential $\mu=-U / 2$ in the BEC limit, and inserting this in the number equation gives a critical temperature

$$
T_{c}=\frac{2 \pi}{[2 \zeta(3 / 2)]^{2 / 3}} n^{2 / 3} J \simeq 2.09 n^{2 / 3} J,
$$


when $n \ll 1$. For $n=1$ we obtain $T_{c}=1.71 J$.

The crossover in optical lattices is shown in Fig. (10) at half filling. The critical temperature decreases with interaction strength in the BEC limit and thus differs from uniform systems where it approaches a constant $T_{c}^{B E C}$.

The Hubbard model can in the BEC limit be mapped onto the Heisenberg spin model in a magnetic field for which accurate calculations exist, which allows us to check the NSR and other models [79]. Half filling corresponds precisely to zero magnetic field, i.e.,

$$
H=J \sum_{\langle i j\rangle} \mathbf{S}_{i} \cdot \mathbf{S}_{j},
$$

and the Néel temperature corresponds to $T_{N}=T_{c}$ in the BEC limit. In (Weiss molecular) mean field theory $T_{N}=1.5 \mathrm{~J}$, quantum Monte Carlo $T_{N}=0.95 \mathrm{~J}$, high temperature expansions $T_{N}=0.90 \mathrm{~J}$, as compared to $T_{c}=1.71 \mathrm{~J}$ in NSR. Extending the NSR model with charge and spin density fluctuations gives a smaller value $T_{c} \simeq 0.4 \mathrm{~J}$ because molecular repulsion is overestimated [79].

\subsection{On-site repulsion}

On-site repulsive interactions $U>0$ generally disfavours doubly occupied sites and lead to a Mott gap at half filling of order $\sim U / 2$ at very low temperatures. Mott insulator (MI) transitions are observed for bosons in traps at fillings $n=1,2,3,4,5, .$. [16]. For fermions the phase diagram is more complex, an antiferromagnet (AF) at and near half filling, a paramagnet (PM) otherwise and possible a ferromagnet for very strong repulsion [86]. Furthermore the AF phase may be unstable towards phase separation to two coexisting phases: an AF at half filling and a PM at filling slightly below or above half filling. The co-existing phase may also be unstable towards stripes. In 2D these phase also compete with d-wave superfluidity.

\subsubsection{Antiferromagnetism}

At half filling fermions are known to form a MI in 1D [80] whereas in two and higher dimensions an antiferromagnetic (AF) insulator is found in Monte-Carlo calculations. The AF alternating spin order on the lattice is driven by the simple fact that hopping can occur to a neighbouring site only if it is occupied by an opposite spin, which generates a super-exchange coupling.

We will here study the AF phase and its transition to a paramagnet at the Néel temperature $T_{N}$. Remarkably, it undergoes a crossover very similar to the critical temperature described above for attractive interactions, i.e. $T_{N} \sim T_{c}$. For weak repulsion $T_{N}$ can be determined from the mean field gap equation 


$$
1=\frac{U}{M} \sum_{\mathbf{p}} \frac{\tanh \left(\varepsilon_{\mathbf{p}} / 2 T_{N}\right)}{2 \varepsilon_{\mathbf{p}}}
$$

which is identical to that for $T_{c}$ of Eq. (71) except for the sign of $U$. Therefore $T_{N}=$ $T_{c}$ within mean field. Induced interactions can be included as for $T_{c}$ as described above and therefore reduce $T_{N}$ by a factor $\sim 3.7$ at half filling [81] which is very similar to the reduction of $T_{c}$ by induced interactions as discussed above.

For stronger coupling fluctuations reduce the mean field gap and $T_{N}$ as for the NSR model of $T_{c}$. At very strong coupling the repulsive Hubbard model can at half filling also be mapped onto the Heisenberg model with coupling $J$. Therefore $T_{N}$ is also in this limit given by $T_{C}$ as function of $|U|$, and the details of the crossover [82, 83] are quite similar to the BCS-BEC crossover.

The AF phase transition masks a possible MI phase. By frustrating the system one can, however, remove the AF order and observe the MI transition by a vanishing conductivity or compressibility. Another way to suppress the AF order is to include multi-components which naturally upsets the alternating spin up/down order in a two-component AF. The limit of many components actually correspond to a Bose system where MI transitions are found at every integer filling.

\subsubsection{Phase separation}

Mean field theory provides a first impression of the phases competing for the ground state and has the advantage that it is computationally simple as compared to more complicated theories. The MF equations for the Hubbard model are standard and we refer to, e.g., Refs. [74, 84]. The energy densities can be calculated within the Hartree-Fock approximation for the paramagnetic (PM), ferromagnetic (FM), antiferromagnetic (AF) and phase separation (PS).

At low density $n \ll 1$ the ground state is that of a dilute paramagnetic (PM) gas with energy

$$
\varepsilon_{P M}=-4 t n+\left[\frac{\pi}{2} t+\frac{1}{4} U\right] n^{2}+\mathscr{O}\left(n^{3}\right) .
$$

In units where the lattice spacing is unity $(\lambda=1)$ this energy per site is also the energy density, and the density is the site filling fraction.

Near half filling $\varepsilon_{P M}=-(4 / \pi)^{2} t+U / 4$, which becomes positive when the repulsive interaction exceeds $U / t \geq 64 / \pi^{2} \simeq 6.5$. The PM phase is then no longer the ground state. In a state with only one spin the antisymmetry of the wavefunction automatically removes double occupancy and the repulsive term $U n^{2} / 4$ in Eq. (82)

disappears. Such a ferromagnetic (FM) state always has negative energy $\varepsilon_{F M} \leq 0$ for $n \leq 1$ and is a candidate for the ground state. AF, linear AF [85] and stripe phases are other competing candidates. Furthermore phase separation (PS) and mixed phases can occur near half filling. When $U \lesssim 7 t$ the ground state of the 2D MF Hubbard model undergoes transitions from an $\mathrm{AF}$ at half filling to a mixed $\mathrm{AF}+\mathrm{PM}$ phase 
for doping $\delta=1-n$ up to a finite ( $U$ dependent) value $|\delta| \leq \delta_{s}$ where after a pure PM phase takes over. [84] For larger $U$ the phase diagram is more complicated with a pure as well as mixed FM phases between the AF and PM phases. A finite next neighbour hopping term $t^{\prime}$ makes the phase diagram asymmetric around half filling, extends the AF phase and changes the phase diagram considerably.

At half filling $n=1$ the ground state is an AF. Near half filling $0 \leq|\delta| \ll 1$ the MF equations and AF energy can be expanded as

$$
\varepsilon_{A F}=-J\left[1+\frac{3}{2} \delta+2 \delta^{2}+\ldots\right]
$$

The concave dependence on $\delta$ signals phase separation into a mixed phase of AF and PM by the Maxwell construction. The PS extends from the AF phase with density $n=1$ to a PM phase. Dynamical mean field theory [86] find that for particle or hole doping $\delta=1-n$, phase separation occurs between an AF at half filling and a paramagnet at a critical filling $\delta_{s}$ depending on the coupling. It has a maximum $\delta_{s} \simeq 0.14$ around the coupling $U \simeq 9 t$ where also $T_{N}$ is maximal. PS is not observed in cuprates. It may be inhibited by long range Coulomb repulsion between electrons in cuprates as will be discussed later.

\subsection{3 d-wave superfluidity in 2D}

d-wave or $d_{x^{2}-y^{2}}$ pairing is particular favoured on a square lattice with on-site repulsion (which disfavours s-wave pairing) and nearest neighbour attraction as e.g. provided by the super-exchange coupling in the Hubbard and Heisenberg model with two spin components. The discussion below emphasizes how 2D and also 3D optical lattices can resolve important issues as the existence of phase separation, stripe phases and dSF.

Repulsive onsite interactions $U>0$ inhibit s-wave pairing unless a longer range attraction is added such as a nearest neighbour interaction $V \sum_{\langle i j\rangle} n_{i} n_{j}$. Superexchange in the Hubbard model generates a similar nearest neighbour (spin-spin) interaction with coupling $J=4 t^{2} / U$, which is believed to be responsible for high temperature superconductivity [87]. In the limit $U \rightarrow \infty$ extended s-wave pairing can occur but requires a strong nearest neighbour attraction $V<-\pi^{2} t / 2$ [74, 88], whereas d-wave pairing occurs naturally in $2 \mathrm{D}$ for even weak nearest neighbour attraction. The d-wave mean field gap equation is (see, e.g., [74, 88])

$$
1=-\frac{V}{4} \sum_{\mathbf{q}} \frac{\eta_{\mathbf{q}}^{2}}{2 E_{\mathbf{q}}},
$$

where now $E_{\mathbf{q}}=\sqrt{\varepsilon_{\mathbf{q}}^{2}+\Delta_{d}^{2} \eta_{\mathbf{q}}^{2}}$ and $\eta_{\mathbf{q}}=2\left[\cos q_{x}-\cos q_{y}\right]$. At low filling the d-wave gap can be calculated within mean field to leading orders in the density [90] 


$$
\Delta_{d}=\frac{t}{\sqrt{n}} \exp \left[\frac{4}{\pi n^{2}}\left(\frac{t}{V}+c_{0}+c_{1} n+c_{2} n^{2}\right)\right], \quad n \ll 1,
$$

The higher order corrections in density are: $c_{0}=4 / \pi-1 \simeq 0.27, c_{1}=\pi / 2-1 \simeq$ 0.57 and $c_{2} \simeq 0.09$.

At half filling we can calculate the d-wave gap within mean field as above if correlations can be ignored, which requires that the on-site interaction is small. To leading logarithmic orders the d-wave pairing gap is

$$
\Delta_{d}=\frac{8}{e^{2}} t \exp [-\pi \sqrt{t /|V|}], \quad n=1
$$

where again the singular level density leads to a gap similar to Eq. (75). It is a coincidence that the prefactor $8 / e^{2}$ is the same as in Eq. (73). Correlations are, however, expected to suppress the d-wave mean field gap of Eq. 86 near half filling when the on-site repulsion is strong.

$T_{C}$ can in 3D be calculated from the gap equation (71) whereas in $2 \mathrm{D}$ a BerezinskiiKosterlitz-Thouless transition occurs [92] at a lower temperature $T_{B K T}$. At low density the transition temperature is in $3 \mathrm{D}$ generally proportional to the gap with the same prefactor $T_{c} / \Delta=e^{C_{E}} / \pi \simeq 0.567$ for both s- and d-wave superfluidity with and without induced interactions [28]. In 2D a mean field critical temperature $T_{M F}>T_{B K T}$ [92], can be calculated from the gap equation as above and we find that the same ratio applies even near half filling where logarithmic singularities appear. This implies that induced interactions change the mean field critical temperatures by the same factor as the gap of Eq. (?).

\subsection{4 t-J-U model predictions}

The various competing phases can be studied in the $2 \mathrm{D}$ t-J-U model within the Gutzwiller projection method and renormalized mean field theory (RMFT) [90]. This method approximates the strong correlations and generally agrees well with full variational Monte Carlo calculations [93, 94]. RMFT predicts phase separation near half filling between an antiferromagnetic (AF) Neél order and a d-wave superfluid (dSF) phase for sufficiently strong onsite repulsion.

The t-J-U model was employed by Laughlin, Zhang and coworkers [94] to study AF, HTc and "gossamer superconductivity" in cuprates and organic superconductors. Both the Hubbard and t-J models are included in the t-J-U Hamiltonian $H=H_{U}+H_{t}+H_{s}$ or

$$
H=U \sum_{i} \hat{n}_{i \uparrow} \hat{n}_{i \downarrow}-t \sum_{\langle i j\rangle \sigma} \hat{a}_{i \sigma}^{\dagger} \hat{a}_{j \sigma}+J \sum_{\langle i j\rangle} \mathbf{S}_{i} \mathbf{S}_{j},
$$

where $\hat{a}_{i \sigma}$ is the usual Fermi creation operator, $\sigma=(\uparrow, \downarrow)$ is the two hyperfine states (e.g. $\left(-\frac{9}{2},-\frac{7}{2}\right)$ for ${ }^{40} \mathrm{~K}$ ), $n_{i \sigma}=\hat{a}_{i \sigma}^{\dagger} \hat{a}_{i \sigma}$ the density, $\mathbf{S}_{i}=\sum_{\sigma \sigma^{\prime}} \hat{a}_{i \sigma}^{\dagger} \sigma_{\sigma \sigma^{\prime}} \hat{a}_{i \sigma^{\prime}}$ and $\langle i j\rangle$ 


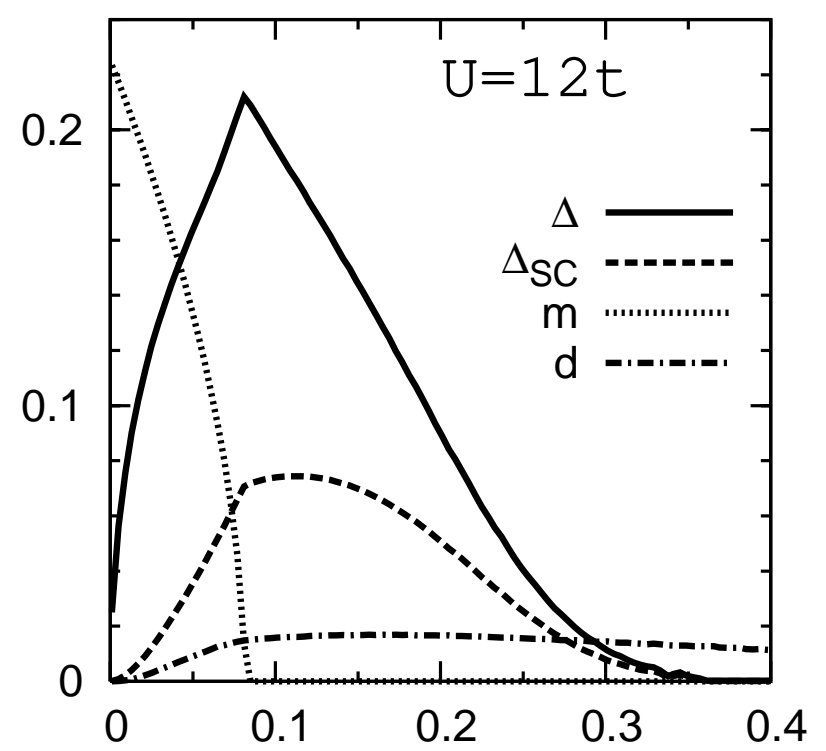

Fig. 11 Order parameters for $\operatorname{dSF}\left(\Delta_{S C}\right), \operatorname{AF}(\mathrm{m})$ and double occupancy (d) vs. doping $\delta=1-n$ for $J / t=4 t / U=1 / 3$ in RMFT for the 2D t-J-U model. From [90].

denotes nearest neighbours. $U$ is the on-site repulsive interaction, $t$ the nearestneighbour hopping parameter and $J$ the spin-spin or super-exchange coupling.

The t-J-U model allows for doubly occupied sites and thereby also MI transitions. As both are observed in optical lattices the t-J-U model is more useful as opposed to the $\mathrm{t}-\mathrm{J}$ model which allows neither. For large $U / t$ the $\mathrm{t}-\mathrm{J}-\mathrm{U}$ and Hubbard models reduce to the t-J model with spin-spin coupling $J=4 t^{2} / U$ due to virtual hopping. At finite $J$ and $U$ the t-J-U model is to some extent double counting with respect to the Hubbard model with $J=0$. However, when RMFT is applied the virtual hopping and resulting spin-spin interaction vanishes in the Hubbard (t-U) model, i.e. $J=0$, which justifies the explicit inclusion of the spin Hamiltonian as done in the t-J-U model.

The AF phase dominates near half filling suppressing the superfluid order parameter. The AF order parameter is $m=(\sqrt{3} / 2) \sqrt{1-T / T_{N}}$ at temperatures just below the critical Nel temperature $T_{N}=2 J$. The d-wave superfluid gap depends sensitively on coupling, magnetization and double occupancy as seen in Fig. (11). It competes with the AF order parameter and dominates slightly away from half filling as seen in Fig. (11).

However, near half filling phase separation is found between an AF phase at half filling coexisting with a dSC phase at a density $|x| \lesssim 0.12$ as determined by the Maxwell construction. The PS is found to terminate at coupling $J \simeq 0.55 t(U \simeq 7.3 t)$ where the double occupancy undergoes a first order transion from zero to a finite 


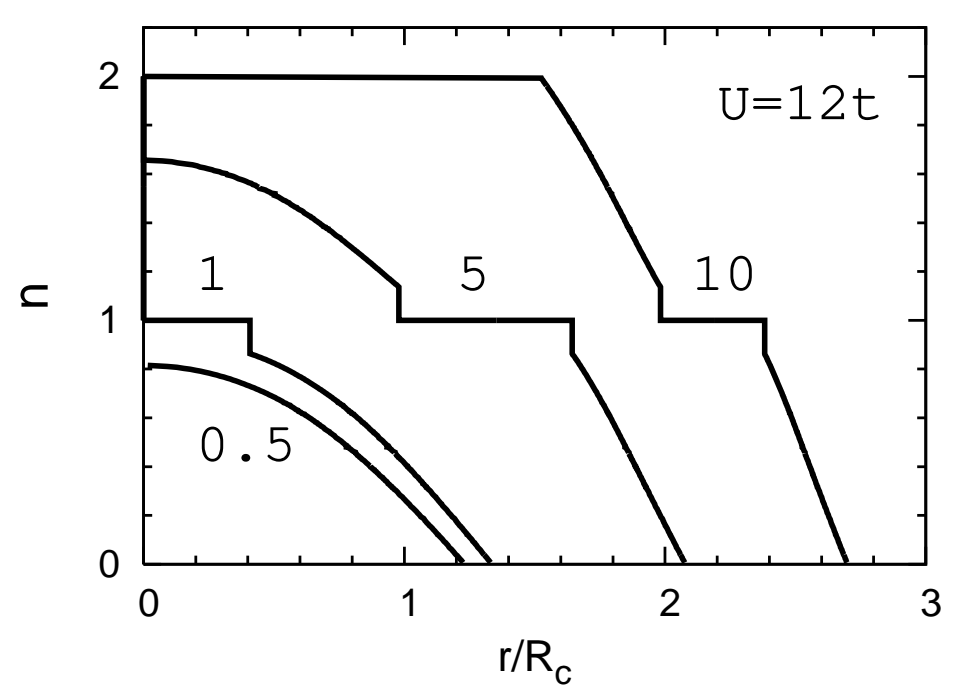

Fig. 12 Density distributions of Fermi atoms in a zero temperature 2D optical lattice confined by a harmonic trap for $U=12 t(\mathrm{~J}=t / 3)$ filled with the number of particles $N / N_{c}=0.5,1,5$, and 10 . The density discontinuities from $n \simeq 1.14$ and $n \simeq 0.86$ to the antiferromagnetic insulator at $n=1$ due to PS between dSF and AF are absent for stripe phases (see text). Thus the density distribution changes to a Capitol form rather than the usual wedding cake form for Mott insulators.

value. The PS has a curious effect on the density distribution in a trap as shown in Fig. (12). The trap size is of order $R_{c}=\sqrt{8 t / \hbar \omega} a_{o s c}$, and the number of trapped particles $N=2 \pi \int_{0}^{R} n(r) r d r$ is of order $N_{c}=\pi R_{c}^{2}$ in $2 \mathrm{D}$.

The density distributions and MI plateaus have been measured experimentally for Bose atoms in optical lattices by, e.g., differentiating between singly and doubly occupied sites [71]. Recently Schneider et al. [89] have measured column densities for Fermi atoms in optical lattices and find evidence for incompressible Mott and band insulator phases. Even lower temperatures are required for observing the $\mathrm{AF}$ and dSF phases and the density discontinuities due to PS.

Spin and charge density waves in form of stripes are not included in the above RMFT calculations. Stripes are observed in several cuprates [100] whereas numerical calculations are model dependent. Long range Coulomb frustration can explain why PS is replaced by stripes and an AF phase at very low doping [90] as observed in cuprates.

\subsection{High temperature superconductivity vs. optical lattices}

After Kamerlingh Onnes discovered superconductivity in 1911 it took nearly half a century before Bardeen, Cooper and Schriefer (BCS) in 1957 understood the physics 
behind the manybody pairing mechanism. In comparison half the time has passed since Bednorz \& Müller in 1986 discovered high temperature superconductivity (HTc). Although important progress has been made on the mechanisms behind this important phenomenon, a full understanding is still lacking. Most agree that HTc is d-wave and can probably be described by the one band Hubbard model on a twodimensional (2D) lattice with strong repulsive on-site interaction $U \sim 3 t$, but no consensus has been reached on the origins and influence of the pseudogap, charge and spin gaps, stripes, etc.

A sketch of a typical phase diagrams of HTc cuprates is shown in Fig. (13). The antiferromagnetic phase extends around half filling and is asymmetric due to next neighbour hopping terms $t^{\prime}$. The Neél temperature drops rapidly from its maximal value $T_{N}(\delta=0)$ - somewhat lower than the Fermi temperature. The maximal $T_{c} \lesssim 100 \mathrm{~K}$ are collected in the "Uemura" plot [99]. It is typically between one and two orders of magnitude lower than the Fermi energy which again is several orders of magnitude larger than conventional superconductors as $\mathrm{Nb}, \mathrm{Sn}, \mathrm{Al}, \mathrm{Zn}$.

Optical lattices hold great promises for solving the HTc problem [95, 96] since densities, temperatures, interactions, spins, etc. can be varied in a controlled manner and studied in experiments, where the sub nanometer lattice constants in solids are upscaled to micrometers in optical lattices. One serious experimental hindrance remains, namely the extremely low temperatures required, but we have hopes that the experimentalists can overcome this problem rapidly as in so many other cases with cold atoms - and it certainly does not hinder theoretical studies.

\subsubsection{Stripes}

Stripes have been discovered in low-energy magnetic neutron scattering in doped cuprates at incommensurate longitudinal and horizontal charge and spin wave numbers [100], e.g. $\mathbf{Q}_{c}=(2 \pi / \lambda)\left(0, \pm \delta / \delta_{s}\right)$ and $\mathbf{Q}_{s}=(\pi / \lambda)\left(1,1 \pm \delta / \delta_{s}\right)$ for the longitudinal charge and spin wave numbers respectively. Here, the average doping or holes are all in the stripes with hole filling $\delta_{s}$ embedded in an AF with filling no holes (half filled) such that the average hole density is $\delta=1-n$. The stripes are half filled $\delta_{s}=1 / 2$ for low average dopings $\delta \leq 1 / 8$ but at larger doping it increases linearly to filled stripes $\delta_{s}=1$ such that $\delta / \delta_{s}=1 / 4$ remains constant. Therefore the charge (spin) density stripes appear with periodic distance $d(2 d)$ depending on doping as $d=\lambda \delta_{s} / \delta$. The stripe distance decreases with increasing doping until $\delta \geq 1 / 8$, where after the stripes remain at a distance $d=4 \lambda$. The stripes act as anti-phase domain walls and the spin density wave therefore has periodicity twice the length of the charge-density wave. When $1 / 8 \leq \delta \leq 1 / 4$ the $2 \mathrm{D}$ spin orientation therefore looks like

$$
\begin{aligned}
& \ldots \downarrow \uparrow \downarrow \circ \uparrow \downarrow \uparrow \circ \downarrow \uparrow \downarrow \circ \uparrow \downarrow \uparrow \circ \ldots \\
& \ldots \uparrow \downarrow \uparrow \circ \downarrow \uparrow \downarrow \circ \uparrow \downarrow \uparrow \circ \downarrow \uparrow \downarrow \circ \ldots \\
& \ldots \downarrow \uparrow \downarrow \circ \uparrow \downarrow \uparrow \circ \downarrow \uparrow \downarrow \circ \uparrow \downarrow \uparrow \circ \ldots
\end{aligned}
$$




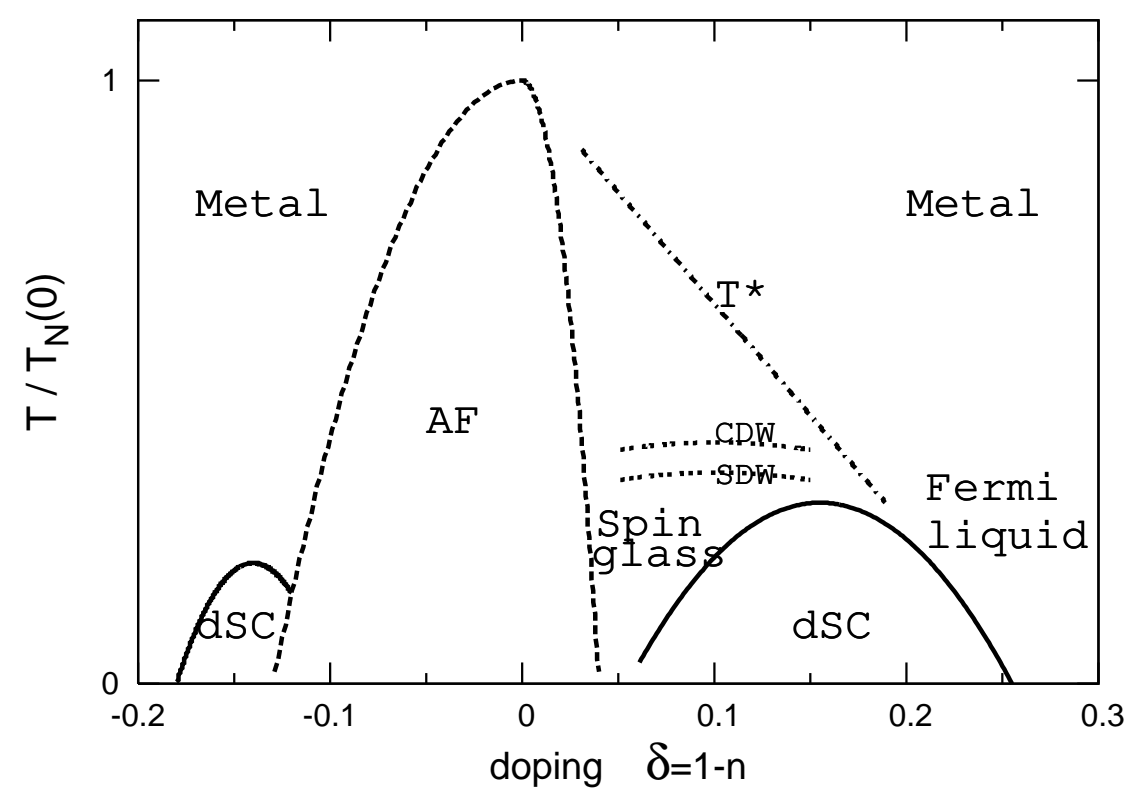

Fig. 13 Typical phase diagram of high temperature superconductors. Neél temperatures $\left(T_{N}\right)$ and critical temperatures for d-wave superconductivity $\left(T_{c}\right)$ are plotted vs. particle $(\delta<0)$ and hole $(\delta>0)$ doping. $T^{*}$ is the pseudogap (see text).

etc., in case of vertical stripes $\circ$. They are spin-balanced at filling $1-\delta_{s}$ and occur with periodicity $d=4 \lambda$.

Diagonal, horizontal/vertical, checkerboard stripe solutions have been found in a number of models. In MF models Zaanen and Gunnarson [97] found stripes of hole density $\delta_{s}=1$ that are vertical or horizontal for $U / t \lesssim 3-4$ and diagonal otherwise. In DMRG calculations [98] stripes with hole density $\delta_{s}=1 / 2$ are found in agreement with experiments [100]. Stripes appear at temperatures above HTc but below the pseudogap $T^{*}[101]$ and there are some evidence that charge density waves appear at slightly higher temperatures than spin density waves.

The stripe phase is a specific ordered mixed AF and PM phase and is a continuous transition between the two pure phases as function of density. Similar mixed phase solutions are believed to occur in neutron star crusts between nuclear matter and a neutron gas and possibly also between quark and nuclear matter [102]. In both cases Coulomb energies add complexity to the mixed phases by ordering them into structured crystallic phases.

The various MI, AF, stripe, d- and s-wave superfluid phases have distinct density and momentum correlation functions [16, 105, 71, 72, 73, 106, 90]. 


\subsubsection{Coulomb frustration}

Long range Coulomb interactions prevent phase separation into two bulk phases of different charge density but may not inhibit the formation of localized holes, pairs and stripes. The phase diagrams of cuprates with such Coulomb frustration and optical lattices without may therefore be very different. Coulomb frustration in cuprates has been discussed in connection with stripes (see e.g. [103, 104]).

In the following we shall consider the stripes as rods with charge less than the surrounding phase and calculate the additional Coulomb energy of such structures. Coulomb energy densities have been calculated for structures of various dimensionality $D$ and volume filling fraction $f$ [102]

$$
\varepsilon_{C}=\frac{2 \pi}{D+2} \Delta \rho^{2} R^{2} f\left[\frac{2}{D-2}\left(1-\frac{D}{2} f^{1-2 / D}\right)+f\right]
$$

Here, the charge density difference between the two phases is $\Delta \rho=e \delta_{s} / \lambda^{3}$ for the stripes and the volume filling fraction is $f=\delta / \delta_{s}=\lambda / d$. The dimensionality is $D=3$ for spherical droplets or bubbles, $D=2$ for rods and tubes and $D=1$ for plate-like structures. The diameter of the spheres, rods or the thickness of the plates is of order the distance between layers $2 R \sim \lambda \sim 4 \AA$. The stripes are rods in a $2 \mathrm{D}$ plane but are embedded in a 3D layered structure. For $D=2$ the expression in the square bracket of Eq. (88) reduces to $[\ln (1 / f)-1+f]$. The logarithm originates from the Coulomb integral $\int^{l} d z / z$ along the rod length $z$, which is cutoff by other rods at a length scale $l \sim \lambda \sqrt{f}$. For the cuprates we furthermore reduce the Coulomb field by a dielectric constant of order $\varepsilon_{D} \sim 5$. The resulting Coulomb energy of stripe or rod-like structures $D=2$ is

$$
\varepsilon_{C} \simeq \frac{\pi}{8} \frac{\delta_{s} e^{2}}{\lambda^{4} \varepsilon_{D}} f[\ln (1 / f)-1+f] .
$$

Energy costs associated with the interface structures are usually added. Such surface energies are difficult to calculate for the stripes because their extent is only a single lattice constant. In principle they are already included in the stripe models. We will therefore just add the Coulomb energies given above. However, the Coulomb energies and the energy of the systems as a whole, may be reduced by screening and hole hopping into the AF whereby $R$ increases but $\Delta \rho$ and $f$ are reduced.

Inserting numbers $e^{2} / \hbar c=1 / 137, \varepsilon_{D}=5, \delta_{s}=1 / 2, \lambda=4 \AA$, we find that $\varepsilon_{c} \simeq$ $150 \mathrm{meV}\left(\delta / \lambda^{3}\right)[\ln (1 / f)-1+f]$. In comparison the energy gain by changing phase from an AF to a stripe or PM phase increases with doping as $\sim J \delta / \lambda^{3}$, where $J \simeq(0.1-0.2) t \simeq 50-100 \mathrm{meV}$ in the cuprates. The Coulomb energy of Eq. 89] thus dominates at small doping due to the logarithmic singularity and therefore the AF phase of density $n \lesssim 1$ is preferred.

The AF phase is the ground state as long as the Coulomb energy of Eq. 89) exceeds $J x / \lambda^{3}$ corresponding to doping less than 


$$
\delta_{A F} \simeq \delta_{s} \exp \left[-\frac{8}{\pi} \frac{J \lambda \varepsilon_{D}}{\delta_{S} e^{2}}-1\right]
$$

Inserting the above numbers we find $\delta_{A F} \simeq 0.1$ which is within range of the observed $\left|\delta_{A F}\right| \simeq 0.03$ for hole doped and $\delta_{A F} \simeq 0.15$ for particle doped cuprates. In MF the particle-hole asymmetry arises from the next-nearest neighbour hopping $t^{\prime} \simeq-0.3 t$ and leads to an AF phase extending from half filling up to a particle doped density $n>1$. 84

In the above picture the incommensurate stripe phases at small doping arise due to Coulomb frustration when $t^{\prime}=0$. At larger doping the stripes approach each other and will eventually affect each other. Experimentally the stripes undergo a transition from an incommensurate to a commensurate phase at $\delta \simeq 1 / 8$ corresponding to a stripe periodicity of four lattice spacings.

The Mermin-Wagner theorem states that a continuous symmetry cannot be spontaneously broken at finite temperature in two and lower dimensional systems for sufficiently short range interactions [91], and as a consequence there can be no phase transition at finite temperature. In stead a Berezinskii-Kosterlitz-Thouless transition can occur as found in the 2D Hubbard model [92]. This is incompatible with the phase diagram of HTc cuprates where AF, dSC and stripe phase transitions are observed. These paradoxial transitions and the origin of the phases are still unresolved problems which are largely avoided in the literature. Some suggest that inter-planar couplings effectively increase the dimension above two. Others that correlations lengths are so long that they effectively looks like real transitions. Another suggestion in line with the Coulomb frustration discussed above is that Coulomb interactions are responsible for the phase transitions to stripes and d-wave superconductivity. The long range Coulomb interactions are not comprised in the Mermin-Wagner theorem, which only applies to sufficiently short range interactions $U(r) \propto r^{-\alpha}$ with $\alpha \geq D+2[91]$.

\section{Summary and Outlook}

In the last decade the physics of cold atoms has brought important understanding of the unitarity limit, universality, the BCS-BEC crossover, and many other properties of cold Fermi atomic systems as describe above and elsewhere in this volume. Presently a number of other interesting phenomena are investigated such as the three component system of ${ }^{6} \mathrm{Li}$ and other multicomponent systems, the ferromagnetic transition at large positive (repulsive) scattering lengths, spin polarized systems, phases in optical lattices, etc.

In these lecture notes the main topic has been universality in crossover. Not only the BCS-BEC crossover in uniform system but also in the repulsive "ferromagnetic" crossover, in multicomponent systems, traps and lattices. The Fermi particles have mostly been atoms but applications to neutron, nuclear and quark matter, nuclei and electrons in solids have been made wherever possible. 
In view on the important impact that the question Bertsch posed a decade ago made, we may attempt to ask new relevant questions:

What are the universal thermodynamic functions and parameters for positive scattering lengths and is there a ferromagnetic phase transition as $a \rightarrow+\infty$ ?

- in both uniform systems and traps. One opinion is given above but a dispute is ongoing [12, 13].

A most promising direction is optical lattices where we may ask:

What are the phase diagrams of the two and three dimensional Hubbard models as realized in optical lattices?

Attractive interactions will lead to a superfluid state with critical temperatures that have been calculated in detail by Monte Carlo. For repulsive interactions the phase diagram is not very well known in $2 \mathrm{D}$ or $3 \mathrm{D}$ even for the single band $\mathrm{t}-\mathrm{U}$ or t-J model. The antiferromagnetic phase may extend to densities near half filling before undergoing a transition to a paramagnet but it is competing with phase separation, stripe phases and a ferromagnetic phase for very strong repulsion. In $2 \mathrm{D}$ it also competes with d-wave superconductivity.

A related question, which is particular relevant for HTc, is:

Does the 2D Hubbard model (as realized in optical lattices) exhibit high temperature superconductivity (as seen in cuprates)?

Because the electrons in HTc have charge they cannot undergo phase separation in bulk, which in turn may be responsible for stripe formation in cuprates in the region of densities and temperatures where also d-wave superconductivity appears. Ultracold Fermi atoms are neutral and can therefore undergo phase separation near half filling as is predicted in some models, e.g. the t-J-U model with Gutzwiller projections discussed above. If so, the region of densities near half filling with antiferromagnetic and d-wave superconductivity do not exist in optical lattices. Cuprates may also have multiband coupling and certainly has next nearest neighbour hoppings, which cause asymmetries in the phase diagrams around half filling for both the AF and dSC phases. In any case, the differences between the phase diagram of the cuprates and the Hubbard model as realized in optical lattices will reveal the important driving mechanisms and possible additional ingredients present in the phenomenon of HTc. 2D optical lattices may also resolve the Mermin-Wagner paradox.

In summary, we have good reasons to believe that ultracold Fermi atoms in traps and optical lattices will in few years bring deeper understanding of strongly correlated systems, its para-, ferro- and antiferromagnetic phases, Mott insulators, stripes, s- and d-wave superfluidity, and high temperature superconductivity. 


\section{References}

1. Proc. of 10th conf. on Recent Progress in Many-Body Theories, World Sci. 1999, Eds. Bishop, R.F., Gernoth, K.A., Walet, N.R., Xian, Y.

2. Baker, G. A., Int. J. Mod. Phys. B 15, 1314 (2001).

3. Heiselberg, H., Phys. Rev. A 63, 043606 (2001).

4. O’Hara, K. M., Hemmer, S. L., Gehm, M. E., Granade, S. R., Thomas, J. E., Science 298 (2002) 2179.

5. Jochim, S., et al., Phys. Rev. Lett. 91, 240402 (2003); Bartenstein, M., et al., ibid. 92, 120401 (2004). Ottenstein, T. B., Lompe, T., Kohnen, M., Wenz, A. N., Jochim, S., Phys. Rev. Lett 101, 203202 (2008).

6. Kitagawa, M., et al., Phys. Rev. A 77, 012719 (2008).

7. Spiegelhalder, F. M., et al., arXiv:0908.1101; Wille, E., et al., Phys. Rev. Lett. 100, 053201 (2008)

8. Alford, M.G., Rajagopal, K., Schaefer, T., and Schmitt, A., Rev. Mod. Phys. 80, 1455 (2008).

9. Eagles, D.M., Phys. Rev. 186, 456 (1969).

10. Leggett, A.J., in Modern Trends in the Theory of Condensed Matter, ed. Pekalski, A., and Przystawa. R., Lect. Notes in Physics, Vol. 115 (Springer-Verlag, 1980), p. 13.

11. Nozières, P., and Schmidt-Rink, S., Low, J., Temp. Phys. 59, 195 (1982). Sá de Melo, C. A. R., Randeria, M., and Engelbrecht, J. R., Phys. Rev. Lett. 71, 3202 (1993).

12. Ho, T.-L., Phys. Rev. Lett. 92, 090402 (2004).

13. Jo, G-B., et al., Science 325, 1521-1524 (2009)

14. Carlson, J., Chang, S-Y., Pandharipande, V. R., Schmidt, K. E., Phys. Rev. Lett. 91, 50401 (2003); Chang, S-Y., et al., Phys. Rev. A 70, 043602, 2004; Nucl. Phys. A 746, 215, 2004.

15. Astrakharchik, G.E., Boronat, J., Casulleras, J., Giorgini, S., Phys. Rev. Lett. 93, 200 (2004).

16. Hadzibabic, Z., Gupta, S., Stan, C. A., Schunck, C. H., Zwierlein, M. W., Dieckmann, K., and Ketterle, W., Phys. Rev. Lett. 91, 160401 (2003). M.W. Zwierlein et al., Phys. Rev. Lett. 91, 250401 (2003); ibid. 92, 120403 (2004). Gupta, S., et al., Science 300, 47 (2003). Chin, J.K. et al., Nature 443, 961 (2006).

17. Bourdel, T., et al., Phys. Rev. Lett. 91, 020402 (2003); cond-mat/0403091; Cubizolles, J., et al., cond-mat/0308018

18. Regal, C. A., Jin, D. S., Phys. Rev. Lett. 90, 230404 (2003); Regal, C. A., Ticknor, C., Bohn, J. L., Jin, D. S., cond-mat/0305028.

19. Chin, C., Bartenstein, M., Altmeyer, A., Riedl, S., Jochim, S., Hecker Denschlag, J., Grimm, R., Science 305, 1128 (2004); Bartenstein, M., et al., Phys. Rev. Lett. 92, 203201 (2004).

20. Nascimbene, S., Navon, N., Jiang, K.J., Cevy, F., Salomon, C., arXiv:0911.0747

21. Heiselberg, H., J. Phys. B: At. Mol. Opt. Phys. 37 (2004) 1-13

22. Cowell, S., Heiselberg, H., Mazets, I. E., Morales, J., Pandharipande, V. R., and Pethick, C. J., Phys. Rev. Lett. 88, 210403 (2002).

23. Holland, M. J., DeMarco, B., and Jin, D. S., Science, 285, 1703 (1999); DeMarco, B., Papp, S. B., and Jin, D. S., Phys. Rev. Lett. 86, 5409 (2001). Truscott, S. G., et al., Science 291, 2570 (2001). Schreck, F., et al, Phys. Rev. A 64, 011402 (2001) O’Hara, K. M., et al., Phys. Rev. Lett. 85, 2092 (2000)

24. Lee, T.D. and Yang, C.N., Phys. Rev. 105, 1119 (1957); Belyakov, V.A., Sov. Phys. JETP 13, 850 (1961).

25. Heiselberg. H., Phys. Rev. A 73, 013607 (2006)

26. Yu, Z., Bruun, G.M., Baym, G., Phys. Rev. A 80, 023615 (2009).

27. Griffin, A, and Zaremba, E., Phys. Rev. A 956, 4839 (1997).

28. Pethick, C. J., Smith, H., Bose-Einstein Condensation in Dilute Gases, Cambridge Univ. Press (2002).

29. Pandharipande, V. R., Nucl. Phys. A 174, 641 (1971) and 178, 123 (1971); Pandharipande, V. R., and Bethe, H. A., Phys. Rev. C 7, 1312 (1973); Pandharipande, V. R., and Schmidt, K. E., Phys. Rev. A 15, 2486 (1977).

30. Carlson, J., Heiselberg, H., and Pandharipande, V. R., Phys. Rev. C 63, 017603 (2001). 
31. Heiselberg, H., Phys. Rev. A 68, 053616 (2003)

32. Strecker, K. E., Partridge, G. B., Hulet, R. G., Phys. Rev. Lett. 91, 080406 (2003). Partridge, G.B. et al., Phys. Rev. Lett. 95, 020404 (2005).

33. Werner, F., Tarruell, L., Castin, Y., Eur. Phys. J. B 68 (2009) 401

34. Fisher, M., Phil. Mag. 7, 1731 (1962)

35. Tan, S., Annals of Physics 323 (2008) 2971-2986

36. Houbiers, M., et al., Phys. Rev. A 56, 4846 (1997).

37. Pandharipande, V. R., priv. comm., Nordita 1999.

38. Brueckner, K. A., Levinson, C. A., and Mahmoud, H. M., Phys. Rev. 103, 1353 (1956); Bethe, H. A., and Goldstone, J., Proc. Roy. Soc. (London), A238, 551 (1957).

39. Baym, G., and Pethick, C. J., Ann. Rev. Astr. \& Astrophysics 17, 415 (1979). Pandharipande, V. R., Int. J. of Mod. Phys. B13, 543 (1999). Heiselberg, H., and Pandharipande, V. R., Ann. Rev. Nucl. \& Part. Science 50 (2000) 481-524. Heiselberg, H., and Hjorth-Jensen, M., Phys. Rep. 50 (2000) 481-524.

40. Heiselberg, H., Pethick, C. J., Ravenhall, D. G., Phys. Rev. Letts. 61, 818 (1988).

41. Efimov, V., Phys. Lett. B33, 563 (1970)

42. Braaten, E., et al., Phys.Rev.Lett. 87 (2001) 160407; Phys.Rev. A 63 (2001) 063609

43. Jonsell, S., Heiselberg, H., Pethick, C. J., Phys. Rev. Lett. 88 (2002) 50401.

44. Stoner, E., Phil. Mag. 15, 1018 (1933).

45. Conduit, G.J., Simons, B.D., arXiv: 0907.372

46. Zhai, H., arXiv: 0909.4917.

47. Bardeen, J., Cooper, L. N., Schrieffer, J. R., Phys. Rev. 108, 1175 (1957).

48. Bogoliubov, N.N., Sov. Phys. JETP 7, 41 (1958).

49. van Hove, L., Physica 25, 849 (1959); Emery, V.J., Nucl. Phys. 19, 154 (1960).

50. Gorkov, L. P., \& Melik-Barkhudarov, T. K., JETP 13, 1018 (1961);

51. Heiselberg, H., Pethick, C. J., Smith, H., and Viverit, L., Phys. Rev. Letts. 85, 2418 (2000).

52. Martikainen, J.-P., Kinnunen, J., Törma, P., and Pethick, C.J., Phys. Rev. Lett. 103, 260403 (2009)

53. Kim, D.-H., Törma, P., and Martikainen, J.-P., Phys. Rev. Lett. 102, 245301 (2009). Heiselberg, H., arXiv:0905.2107

54. Petrov, D.S., Salomon, C., and Shlyapnikov, G., Phys. Rev. Lett. 93, 090404 (2004).

55. Schirotzek, A., Shin, Y., Schunck, C.H., Ketterle, W., arXiv:0808.0026

56. Pieri, P., Pisani, L., and Strinati, G.C., Phys. Rev. B 70, 094508 (2004).

57. Bloch, I., Dalibard, J., and Zwerger, W., Rev. Mod. Phys. 80, 885 (2008).

58. Haussmann, R., Rantner, W., Cerrito, S., Zwerger, W., Phys. Rev. A 75, 023610 (2007).

59. Bohr, A., and Mottelson, B. R., Nuclear Structure Vols. I+II, Benjamin, New York 1969.

60. Heiselberg, H., Phys. Rev. Lett. 93, 040402 (2004).

61. Cozzini, M., Stringari, S., Phys. Rev. A 67, 041602 (R) (2003).

62. Riedl, S., et al., arXiv:0907.3814

63. Clancy, B., Luo, L., Thomas, J.E., arXiv:0705.2782

64. Bohr, A., Mottelson, B. R., Pines, D., Phys. Rev. 110, 936 (1958).

65. Heiselberg, H., and Mottelson, B. R., Phys. Rev. Lett. 88, 190401 (2002). Bruun, G. M., and Heiselberg, H., Phys. Rev. A 65, 053407 (2002).

66. Dobaczewski, J., et al., Phys.Rev. C63, 024308 (2001).

67. Audi, G., and Wapstra, A. H., Nucl. Phys. A595, 409 (1995). Dobaczewski, J., et al., Phys.Rev. C63, 024308 (2001).

68. Baym, G., Heiselberg, H., Phys.Rev. D 56 (1997) 5254-5259

69. Thomas, J. E., proc. of Quark Matter 09; Gelman, B. A., Shuryak, E. V., Zahed, I., Phys. Rev. A 72 043601,2005. Schaefer, T. and Teaney, D., Rept. Prog. Phys. 72 126001, 2009

70. Baym, G., ActaPhys.Polon. B29:1839-1884,1998; Heiselberg, H., Phys. Rev. Lett. 82 (1999) 2052-2055

71. Fölling, S., et al., Nature 434, 481 (2005).

72. Spielman, I. B., Phillips, W. D., and Porto, J. V., Phys. Rev. Lett. 98, 080404 (2007).

73. Rom, T., Best, Th., van Oosten, D., Schneider, U., Fölling, S., Paredes, B., Bloch, I., Nature 444, 733 (2006). 
74. Micnas, R., Ranninger, J., Robaszkiewicz, S., and Tabor, S., Phys. Rev. B 37, 9410 (1988); Rev. Mod. Phys. 67, 117 (1998)

75. Marsiglio, F., Phys. Rev. B 55, 575 (1997).

76. Burovski, E., Prokofev, N., Svistunov, B., Troyer, M., Phys. Rev. Lett. 96, 160402 (2006). Burovski, E., Kozik, E., Prokof'ev, N., Svistunov, B., Troyer, M. Phys. Rev. Lett. 101, 090402 (2008).

77. Dupuis, N., Phys. Rev. B 70, 134502 (2004). Note that the approximate level density used leads to a gap reduced by a factor $2 / \pi$ in the dilute limit.

78. Nishida, Y. \& Son, D. T., Phys. Rev. A 75063617 (2007).

79. Tamaki, H., Ohashi, Y., Miyake, K., arXiv:0803.2084

80. Lieb, E. H., and Wu, F. Y., Phys. Rev. Lett. 20, 1445 (1968).

81. van Dongen, P.G.J., Phys. Rev. Lett. 67, 757 (1991); Phys. Rev. B 50, 14016 (1994).

82. Staudt, R., Dzierzawa, M., Muramatsu, A., Eur. Phys. J. B 17, 411 (2000)

83. Georges, A., arXiv:cond-mat/0702122.

84. Langmann, E., and Wallin, M., cond-mat/0406608.

85. Kusko, C., and Markiewicz, R. S., cond-mat/0102440.

86. Pruschke, T. and Zitzler, R., J. Phys.: Condens. Matter 15, 7867 (2003).

87. Anderson, P. W., Science 235, 1196 (1987).

88. Pistolesi, F., and Nozieres, Ph., Phys. Rev. B 66, 054501 (2002).

89. Schneider, U., et al., Science 322, 1520 (2008)

90. Heiselberg, H., Phys. Rev. A 79, 063611 (2009).

91. Bogoliubov, N.N., Physik Abhandl. Sowjetunion 6, 1 (1962). Mermin, N.D. and Wagner, H., Phys. Rev. Lett. 17, 1133 (1966).

92. Paiva, T., Scalettar, R., Randeria, M., and Trivedi, N., arXiv:0906.2141.

93. See e.g., Edegger, B., Muthukumar, V. N., Gros, C., Advances in Physics 56, 927 (2007), and Refs. therein.

94. Laughlin, R. B., arXiv:cond-mat/0209269; Zhang, F. C., Phys. Rev. Lett. 90, 207002 (2003); Gan, J. Y., Zhang, F. C., Su, Z. B., Phys. Rev. B 71, 014508 (2005).

95. Hofstetter, W., Cirac, J. I., Zoller, P., Demler, E., and Lukin, M. D., Phys. Rev. Lett. 89, 220407 (2002).

96. Chen et al., Phys. Rep. 412, 1 (2005).

97. Zaanen, J., and Gunnarsson, O., Phys. Rev. B 40, 7391 (1989). Poilblanc, D., and Rice, T. M., Phys. Rev. B 39, 9749 (1989). Schulz, H. J., J. Physique, 50, 2833 (1989); Machida, K., Physica C 158, 192 (1989).

98. Scalapino, D. J., Physics Reports 250, 329 (1995); and cond-mat/0610710, to appear as Chapter 13 in "Handbook of High Temperature Superconductivity", Schrieffer, J. R., editor, Springer, 2006. White, S. R., and Scalapino, D. J., Phys. Rev. Lett. 81, 3227 (1998).

99. Uemura, Y.J., Physica C 282, 194 (1997).

100. Tranquada, J. M., et al., Phys. Rev. B 54, 7489 (1996).

101. Berg, E., Fradkin, E., Kivelson, S.A., Tranquada, J., New J. Phys 11, 115004 (2009). Vojta, M., Adv. Phys. 58, 699 (2009)

102. Pethick, C. J., \& Ravenhall, G. R., Ann. NY Acad. Sci. 647, 503 (1991). Heiselberg, H., Pethick, C.J., Staubo, E.F., Phys. Rev. Lett. 70, 1355 (1993).

103. Seibold, G., Castellani, C., Di Castro, C., and Grilli, M., cond-mat/9803184. Lorenzana, J., Castellani, C., Di Castro, C., Phys. Rev. B 64, 235128 (2001).

104. Arrigoni, E., Fradkin, E., and Kivelson, S. A., Phys. Rev. B 69, 214519 (2004).

105. Köhl, M., Moritz, H., Stöferle, T., Gunter, K., Esslinger, T., Phys. Rev. Lett. 94, 080403 (2005).

106. Andersen, B. M. and Bruun, G. M., Phys. Rev. A 76, 041602(R) (2007) 